Supplementary Information

\title{
Metal-free Direct Hydrosulfonylation of Azodicarboxylates with Sulfinic acids Leading to Sulfonylhydrazine Derivatives
}

\author{
Jiangwei Wen,Wei Wei, ${ }^{*}$ Daoshan Yang, Yufeng Fan, Lulu Fu, Hua Wang*
}

The Key Laboratory of Life-Organic Analysis and Key Laboratory of Pharmaceutical Intermediates and Analysis of Natural Medicine, School of Chemistry and Chemical Engineering, Qufu Normal University, Qufu 273165, Shandong, China.

"Phone: +86 537 4458317; Fax: +86 537 4458317. E-mail: weiweiqfnu@163.com; huawang_qfnu@126.com

\section{Contents}

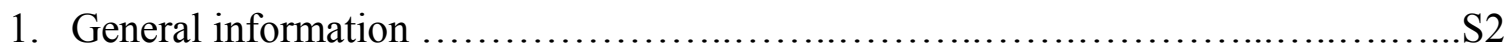

2. General procedure for metal-free direct hydrosulfonylation of azodicarboxylates with

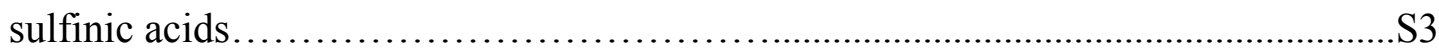

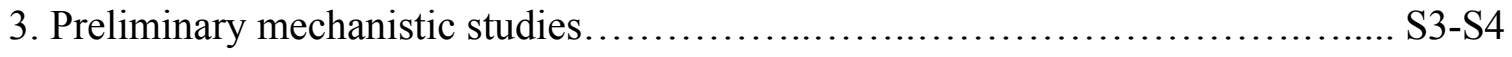

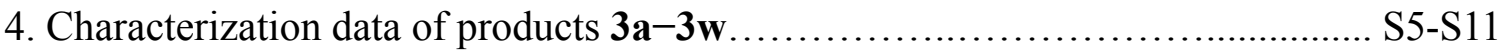

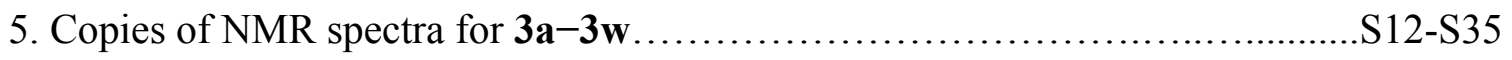




\section{General information}

All commercially available reagent grade chemicals were purchased from Aldrich, Acros, Alfa Aesar and Beijing Ouhe Chemical Company and used as received without further purification unless otherwise stated. All solvents were dried according to standard procedures. ${ }^{1} \mathrm{H}$ NMR and ${ }^{13} \mathrm{C}$ NMR were recorded in $\mathrm{CDCl}_{3}$ on a Bruker Avance III 400 spectrometer with TMS as internal standard $\left(400 \mathrm{MHz}{ }^{1} \mathrm{H}, 100 \mathrm{MHz}{ }^{13} \mathrm{C}\right)$ at room temperature, the chemical shifts $(\delta)$ were expressed in ppm and $J$ values were given in $\mathrm{Hz}$. The following abbreviations are used to indicate the multiplicity: singlet (s), doublet (d), triplet (t), quartet (q), doublet of doublets (dd), doublet of triplets (dt), and multiplet (m). All first order splitting patterns were assigned on the basis of the appearance of the multiplet. Splitting patterns that could not be easily interpreted were designated as multiplet (m). Mass analyses and HRMS were obtained on a Finnigan-LCQDECA mass spectrometer and a Bruker Daltonics Bio-TOF-Q mass spectrometer by the ESI method, respectively. Column chromatography was performed on silica gel (200-300 mesh). 


\section{General procedure for metal-free direct hydrosulfonylation of azodicarboxylates with sulfinic acids}

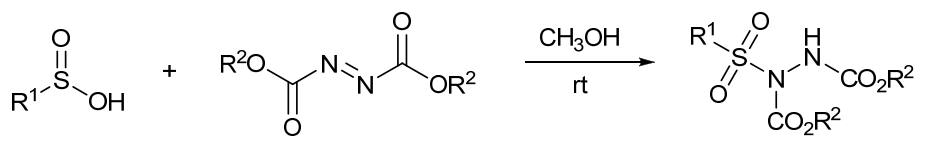

To a mixture of sulfinic acids $1(0.6 \mathrm{mmol})$ and azodicarboxylates $2(0.5 \mathrm{mmol})$ in a $25 \mathrm{~mL}$ round-bottomed flack at room temperature, was added the $\mathrm{CH}_{3} \mathrm{OH}(2 \mathrm{~mL})$. The reaction vessel was allowed to stir at room temperature for $12 \mathrm{~h}$. After the reaction, the solvent was then removed under vacuum. The residue was purified by flash column chromatography using a mixture of petroleum ether and ethyl acetate as eluent to give the desired product 3 .

\section{Preliminary mechanistic studies.}

(1) The reaction of benzenesulfinic acid 1a and diisopropyl azodicarboxylate $\mathbf{2 a}$ in the presence of TEMPO or BHT.

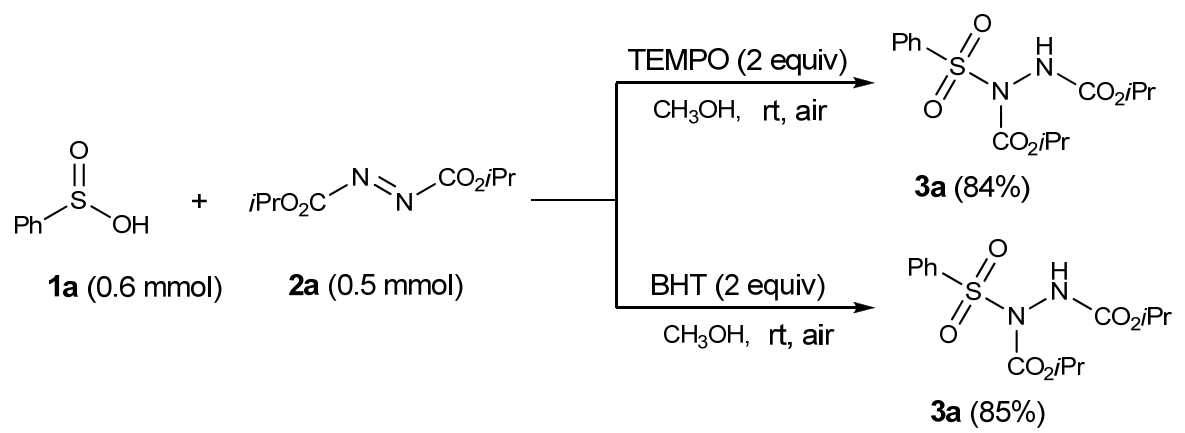

To a mixture of benzenesulfinic acid $\mathbf{1 a}(0.6 \mathrm{mmol})$, diisopropyl azodicarboxylate $\mathbf{2 a}$ $(0.5 \mathrm{mmol})$ and TEMPO $(1.0 \mathrm{mmol})$ or BHT $(1.0 \mathrm{mmol})$ in a $25 \mathrm{~mL}$ round-bottomed flack at room temperature, was added the $\mathrm{CH}_{3} \mathrm{OH}(2 \mathrm{~mL})$. The reaction vessel was allowed to stir at room temperature for $12 \mathrm{~h}$. After the reaction, the solution was concentrated in vacuum, the corresponding product 3a were obtained in $84 \%$ and $85 \%$ yields, respectively.

(2) The reaction of benzenesulfonic acid with diisopropyl azodicarboxylate $\mathbf{2 a}$. 


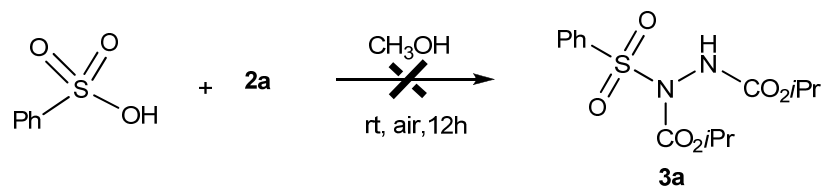

To a mixture of benzenesulfonic acid $(0.6 \mathrm{mmol})$ and diisopropyl azodicarboxylate $2 \mathbf{a}$ $(0.5 \mathrm{mmol})$ in a $25 \mathrm{~mL}$ round-bottomed flack at room temperature, was added the $\mathrm{CH}_{3} \mathrm{OH}$ $(2 \mathrm{~mL})$. The reaction vessel was allowed to stir at room temperature for $12 \mathrm{~h}$. After the reaction, the solution was concentrated in vacuum, the corresponding product 3a was not detected.

(3) The reaction of sodium benzenesulfinate with diisopropyl azodicarboxylate $\mathbf{2 a}$.

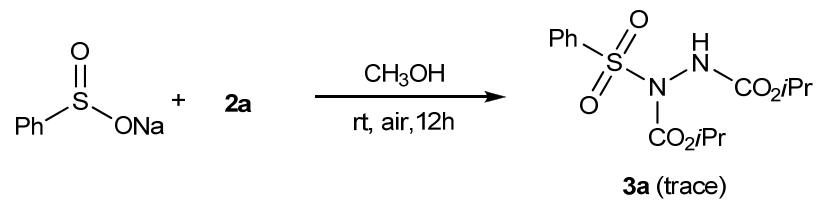

To a mixture of sodium benzenesulfinate $(0.6 \mathrm{mmol})$ and diisopropyl azodicarboxylate $2 \mathrm{a}(0.5 \mathrm{mmol})$ in a $25 \mathrm{~mL}$ round-bottomed flack at room temperature, was added the $\mathrm{CH}_{3} \mathrm{OH}(2 \mathrm{~mL})$. The reaction vessel was allowed to stir at room temperature for $12 \mathrm{~h}$. After the reaction, the solution was concentrated in vacuum, only a trace amount of corresponding product 3a was not detected.

(4) The reaction of sodium benzenesulfinate with diisopropyl azodicarboxylate $\mathbf{2 a}$.

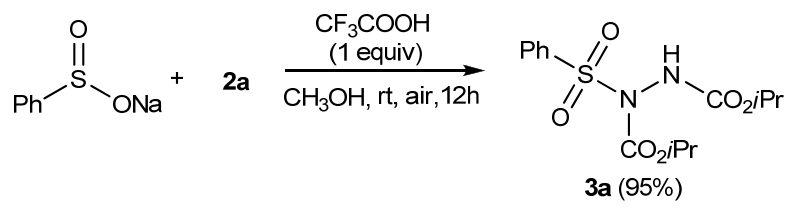

To a mixture of sodium benzenesulfinate $(0.6 \mathrm{mmol})$, diisopropyl azodicarboxylate 2a $(0.5 \mathrm{mmol})$ and $\mathrm{CF}_{3} \mathrm{COOH}(0.5 \mathrm{mmol})$ in a $25 \mathrm{~mL}$ round-bottomed flack at room temperature, was added the $\mathrm{CH}_{3} \mathrm{OH}(2 \mathrm{~mL})$. The reaction vessel was allowed to stir at room temperature for $12 \mathrm{~h}$. After the reaction, the solution was concentrated in vacuum, the corresponding product 3a was isolated in $95 \%$ yield. 


\section{Characterization data of products 3a-3w}

diisopropyl 1-(phenylsulfonyl)hydrazine-1,2-dicarboxylate

Compound 3a was obtained in 98\% yield according to the general procedure (12h).

${ }^{1} \mathrm{H}$ NMR $\left(\mathrm{CDCl}_{3}, 400 \mathrm{MHz}, \mathrm{ppm}\right): \delta 8.15$ (d, $\left.J=7.6 \mathrm{~Hz}, 2 \mathrm{H}\right), 7.65$ (t, $\left.J=7.3 \mathrm{~Hz}, 1 \mathrm{H}\right)$, $7.54(\mathrm{t}, J=8.0 \mathrm{~Hz}, 2 \mathrm{H}), 7.17$ (brs, 1H), 5.01-4.91 (m, 2H), 1.33-1.11(m, 12H); ${ }^{13} \mathrm{C} \mathrm{NMR}$ $\left(\mathrm{CDCl}_{3}, 100 \mathrm{MHz}, \mathrm{ppm}\right):$ 154.6, 150.9, 138.3, 134.0, 129.4, 128.6, 73.0, 71.0, 21.9, 21.5. HRMS (ESI) calcd for $\mathrm{C}_{14} \mathrm{H}_{20} \mathrm{~N}_{2} \mathrm{O}_{6} \mathrm{NaS}(\mathrm{M}+\mathrm{Na})^{+}$367.0940, found 367.0945.

\section{Diisopropyl1-(4-methoxyphenylsulfonyl)hydrazine-1,2-dicarboxylate}

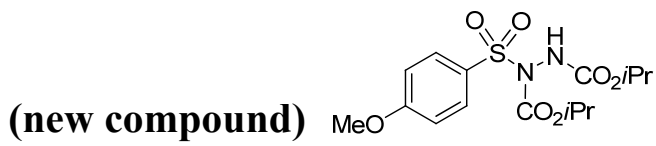

(3b)

Compound $\mathbf{3 b}$ was obtained in $97 \%$ yield according to the general procedure (12h).

${ }^{1} \mathrm{H}$ NMR $\left(\mathrm{CDCl}_{3}, 400 \mathrm{MHz}, \mathrm{ppm}\right): \delta 8.06(\mathrm{~d}, J=8.5 \mathrm{~Hz}, 2 \mathrm{H}), 7.04$ (brs, 1H), 7.01 (d, $J=$ $9.0 \mathrm{~Hz}, 2 \mathrm{H}), 5.00-4.93(\mathrm{~m}, 2 \mathrm{H}), 3.90(\mathrm{~s}, 3 \mathrm{H}), 1.34-1.17(\mathrm{~m}, 12 \mathrm{H}) ;{ }^{13} \mathrm{C} \mathrm{NMR}\left(\mathrm{CDCl}_{3}, 100\right.$ MHz, ppm): 164.1, 154.5, 151.0, 131.9, 129.5, 113.8, 72.8, 70.9, 55.7, 21.9, 21.6. HRMS (ESI) calcd for $\mathrm{C}_{15} \mathrm{H}_{22} \mathrm{~N}_{2} \mathrm{O}_{7} \mathrm{NaS}(\mathrm{M}+\mathrm{Na})^{+} 397.1045$, found 397.1049.

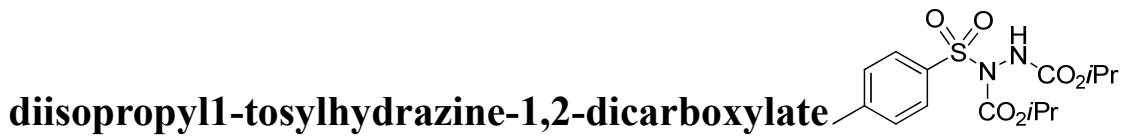

Compound $3 \mathbf{c}$ was obtained in $86 \%$ yield according to the general procedure $(12 \mathrm{~h})$.

${ }^{1} \mathrm{H}$ NMR $\left(\mathrm{CDCl}_{3}, 400 \mathrm{MHz}, \mathrm{ppm}\right): \delta 8.01$ (d, $\left.J=7.8 \mathrm{~Hz}, 2 \mathrm{H}\right), 7.34(\mathrm{~d}, J=8.1 \mathrm{~Hz}, 2 \mathrm{H})$, 7.03 (brs, 1H), 5.01-4.93 (m, 2H), $2.46(\mathrm{~s}, 3 \mathrm{H}), 1.35-1.16(\mathrm{~m}, 12 \mathrm{H}) ;{ }^{13} \mathrm{C} \mathrm{NMR}\left(\mathrm{CDCl}_{3}\right.$, $100 \mathrm{MHz}, \mathrm{ppm}): 154.5,150.9,145.2,135.3,129.5,129.2,72.9,70.9,21.9,21.7,21.6$. HRMS (ESI) calcd for $\mathrm{C}_{15} \mathrm{H}_{22} \mathrm{~N}_{2} \mathrm{O}_{6} \mathrm{NaS}(\mathrm{M}+\mathrm{Na})^{+}$381.1096, found 381.1098.

diisopropyl1-(4-chlorophenylsulfonyl)hydrazine-1,2-dicarboxylate

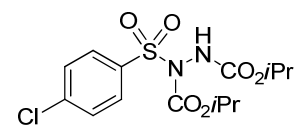
(3d)

Compound 3d was obtained in 98\% yield according to the general procedure (12h).

${ }^{1} \mathrm{H}$ NMR $\left(\mathrm{CDCl}_{3}, 400 \mathrm{MHz}, \mathrm{ppm}\right): \delta 8.09$ (d, $\left.J=8.5 \mathrm{~Hz}, 2 \mathrm{H}\right), 7.53$ (d, $\left.J=8.7 \mathrm{~Hz}, 2 \mathrm{H}\right)$, 7.00 (brs, 1H), 5.01-4.94 (m, 2H), 1.35-1.19 (m, 12H); ${ }^{13} \mathrm{C} \mathrm{NMR}\left(\mathrm{CDCl}_{3}, 100 \mathrm{MHz}\right.$, 
ppm): 154.5, 150.7, 140.8, 136.6, 131.0, 128.9, 73.2, 71.1, 21.9, 21.6; $\mathrm{MS}\left(\mathrm{ESI}^{+}\right)$: $[\mathrm{M}+\mathrm{H}]^{+}$379.1; HRMS (ESI) calcd for $\mathrm{C}_{14} \mathrm{H}_{19} \mathrm{~N}_{2} \mathrm{O}_{6} \mathrm{ClNaS}(\mathrm{M}+\mathrm{Na})^{+} 401.0550$, found 401.0551 .

diisopropyl1-(4-bromophenylsulfonyl)hydrazine-1,2-dicarboxylate (new compound)

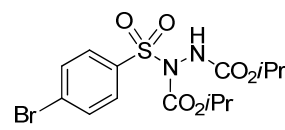

Compound 3e was obtained in $81 \%$ yield according to the general procedure (12h).

${ }^{1} \mathrm{H}$ NMR ( $\left.\mathrm{CDCl}_{3}, 400 \mathrm{MHz}, \mathrm{ppm}\right): \delta 8.01(\mathrm{~d}, J=8.2 \mathrm{~Hz}, 2 \mathrm{H}), 7.69(\mathrm{~d}, J=8.8 \mathrm{~Hz}, 2 \mathrm{H})$, 7.04 (brs, $1 \mathrm{H}), 5.02-4.94(\mathrm{~m}, 2 \mathrm{H}), 1.35-1.18(\mathrm{~m}, 12 \mathrm{H}) ;{ }^{13} \mathrm{C} \mathrm{NMR}\left(\mathrm{CDCl}_{3}, 100 \mathrm{MHz}\right.$,

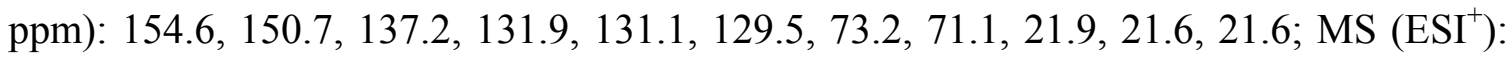
$[\mathrm{M}+\mathrm{H}]^{+}$423.0; HRMS (ESI) calcd for $\mathrm{C}_{14} \mathrm{H}_{19} \mathrm{~N}_{2} \mathrm{O}_{6} \mathrm{BrNaS}(\mathrm{M}+\mathrm{Na})^{+}$445.0045, found 445.0047 .

diisopropyl1-(4-fluorophenylsulfonyl)hydrazine-1,2-dicarboxylate (new compound)

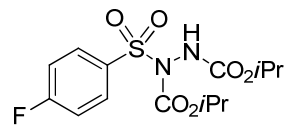

(3f)

Compound $3 \mathrm{f}$ was obtained in $87 \%$ yield according to the general procedure (12h).

${ }^{1} \mathrm{H} \mathrm{NMR}\left(\mathrm{CDCl}_{3}, 400 \mathrm{MHz}, \mathrm{ppm}\right): \delta 8.18\left(\mathrm{dd}, J_{1}=8.2 \mathrm{~Hz}, J_{2}=4.8 \mathrm{~Hz}, 2 \mathrm{H}\right), 7.23$ (t, $J=$ $8.2 \mathrm{~Hz}, 2 \mathrm{H}), 7.06$ (brs, 1H), 5.02-4.92 (m, 2H), 1.35-1.17 (m, 12H); ${ }^{13} \mathrm{C} \mathrm{NMR}\left(\mathrm{CDCl}_{3}\right.$, $100 \mathrm{MHz}, \mathrm{ppm}): 166.0$ (d, $J=255.5 \mathrm{~Hz}), 154.5,150.8,134.1,132.5(\mathrm{~d}, J=9.6 \mathrm{~Hz})$,

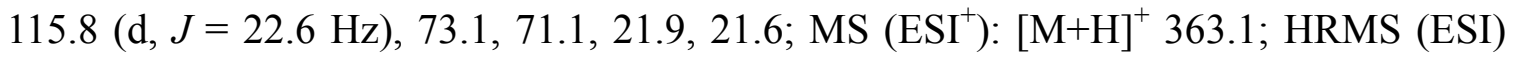
calcd for $\mathrm{C}_{14} \mathrm{H}_{19} \mathrm{~N}_{2} \mathrm{O}_{6} \mathrm{FNaS}(\mathrm{M}+\mathrm{Na})^{+}$385.0846, found 385.0843.

diisopropyl1-(2-bromophenylsulfonyl)hydrazine-1,2-dicarboxylate (new compound)

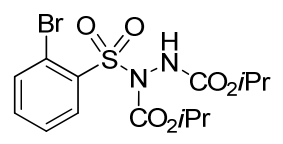

(3g)

Compound $\mathbf{3 g}$ was obtained in $86 \%$ yield according to the general procedure (12h).

${ }^{1} \mathrm{H} \mathrm{NMR}\left(\mathrm{CDCl}_{3}, 400 \mathrm{MHz}, \mathrm{ppm}\right): \delta 8.36$ (brd, $\left.J=6.4 \mathrm{~Hz}, 1 \mathrm{H}\right), 7.76$ (dd, $J_{1}=1.2 \mathrm{~Hz}, J_{2}$ $=7.8 \mathrm{~Hz}, 1 \mathrm{H}), 7.58-7.49(\mathrm{~m}, 2 \mathrm{H}), 7.01(\mathrm{brs}, 1 \mathrm{H}), 5.11-5.05(\mathrm{~m}, 1 \mathrm{H}), 4.96-4.90(\mathrm{~m}, 1 \mathrm{H})$, 1.34-1.32 (m, 6H), $1.24(\mathrm{~d}, J=6.2 \mathrm{~Hz}, 3 \mathrm{H}), 1.07-1.03(\mathrm{~m}, 3 \mathrm{H}) ;{ }^{13} \mathrm{C}$ NMR $\left(\mathrm{CDCl}_{3}, 100\right.$ MHz, ppm): 154.8, 150.6, 138.3, 135.2, 134.7, 133.6, 127.7, 120.0, 73.2, 71.1, 21.9, 21.7, 21.5, 21.3; $\mathrm{MS}\left(\mathrm{ESI}^{+}\right)$: $[\mathrm{M}+\mathrm{H}]^{+}$423.0; HRMS (ESI) calcd for $\mathrm{C}_{14} \mathrm{H}_{19} \mathrm{~N}_{2} \mathrm{O}_{6} \mathrm{BrNaS}(\mathrm{M}+$ 
$\mathrm{Na})^{+} 445.0045$, found 445.0048 .

Diisopropyl1-(4-(trifluoromethyl)phenylsulfonyl)hydrazine-1,2-dicarboxylate (new compound) $\mathrm{F}_{3} \mathrm{C}$

Compound $\mathbf{3 h}$ was obtained in $87 \%$ yield according to the general procedure $(12 \mathrm{~h})$.

${ }^{1} \mathrm{H}$ NMR $\left(\mathrm{CDCl}_{3}, 400 \mathrm{MHz}, \mathrm{ppm}\right): \delta 8.30(\mathrm{~d}, J=8.1 \mathrm{~Hz}, 2 \mathrm{H}), 7.83(\mathrm{~d}, J=8.4 \mathrm{~Hz}, 2 \mathrm{H})$,

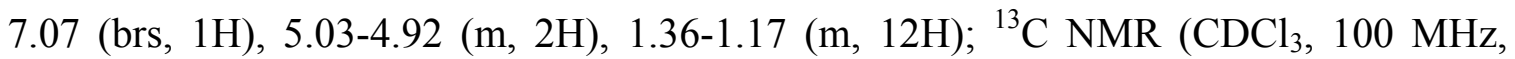
ppm): 154.5, 150.6, 141.7 (d, $J=1.3 \mathrm{~Hz}), 135.5$ (d, $J=33.8 \mathrm{~Hz}), 130.1,125.7,123.1$ (d, $J=271.6 \mathrm{~Hz}), 73.5,71.3,21.8,21.5$; HRMS (ESI) calcd for $\mathrm{C}_{15} \mathrm{H}_{19} \mathrm{~N}_{2} \mathrm{O}_{6} \mathrm{~F}_{3} \mathrm{NaS}(\mathrm{M}+$ $\mathrm{Na})^{+} 435.0814$, found 435.0815 .

diisopropyl1-(4-acetylphenylsulfonyl)hydrazine-1,2-dicarboxylate (new compound)

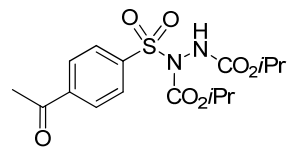

(3i)

Compound $3 \mathbf{i}$ was obtained in $93 \%$ yield according to the general procedure (12h).

${ }^{1} \mathrm{H}$ NMR $\left(\mathrm{CDCl}_{3}, 400 \mathrm{MHz}, \mathrm{ppm}\right): \delta 8.23(\mathrm{~d}, J=8.4 \mathrm{~Hz}, 2 \mathrm{H}), 8.08(\mathrm{~d}, J=8.6 \mathrm{~Hz}, 2 \mathrm{H})$, 7.55 (brs, $1 \mathrm{H}), 4.97-4.90(\mathrm{~m}, 2 \mathrm{H}), 2.65(\mathrm{~s}, 3 \mathrm{H}), 1.32-1.24(\mathrm{~m}, 6 \mathrm{H}), 1.19-1.12(\mathrm{~m}, 6 \mathrm{H}) ;{ }^{13} \mathrm{C}$ NMR $\left(\mathrm{CDCl}_{3}, 100 \mathrm{MHz}, \mathrm{ppm}\right):$ 196.9, 154.6, 150.7, 142.0, 140.8, 129.8, 128.3, 73.3, 71.1, 26.9, 21.9, 21.8, 21.6, 21.5; HRMS (ESI) calcd for $\mathrm{C}_{16} \mathrm{H}_{22} \mathrm{~N}_{2} \mathrm{O}_{7} \mathrm{NaS}(\mathrm{M}+\mathrm{Na})^{+}$ 409.1045, found 409.1041.

diisopropyl1-(4-nitrophenylsulfonyl)hydrazine-1,2-dicarboxylate (new compound)

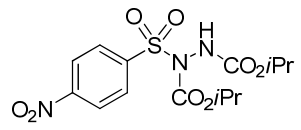

(3j)

Compound $\mathbf{3} \mathbf{j}$ was obtained in $88 \%$ yield according to the general procedure (24h).

${ }^{1} \mathrm{H}$ NMR $\left(\mathrm{CDCl}_{3}, 400 \mathrm{MHz}, \mathrm{ppm}\right): \delta 8.41-8.35$ (m, 4H), 7.18 (brs, $\left.1 \mathrm{H}\right), 5.02-4.93$ (m, $2 \mathrm{H}), 1.35(\mathrm{~d}, J=6.2 \mathrm{~Hz}, 3 \mathrm{H}), 1.30$ (d, $J=6.1 \mathrm{~Hz}, 3 \mathrm{H}), 1.23-1.19(\mathrm{~m}, 6 \mathrm{H}) ;{ }^{13} \mathrm{C} \mathrm{NMR}$ $\left(\mathrm{CDCl}_{3}, 100 \mathrm{MHz}, \mathrm{ppm}\right):$ 154.5, 150.8, 150.5, 143.7, 131.0, 123.7, 73.7, 71.5, 21.8, 21.6, 21.5; HRMS (ESI) calcd for $\mathrm{C}_{14} \mathrm{H}_{19} \mathrm{~N}_{3} \mathrm{O}_{8} \mathrm{NaS}(\mathrm{M}+\mathrm{Na})^{+} 412.0791$, found 412.0790.

Diisopropyl1-(4-acetamidophenylsulfonyl)hydrazine-1,2-dicarboxylate

(new 
compound)

(3k)

Compound 3k was obtained in 97\% yield according to the general procedure $(12 \mathrm{~h}) .{ }^{1} \mathrm{H}$ NMR $\left(\mathrm{CDCl}_{3}, 400 \mathrm{MHz}, \mathrm{ppm}\right): \delta 8.01$ (s, $\left.J=7.7 \mathrm{~Hz}, 2 \mathrm{H}\right), 8.00$ (brs, $\left.1 \mathrm{H}\right), 7.68$ (d, $J=7.6$ $\mathrm{Hz}, 2 \mathrm{H}), 7.30(\mathrm{~s}, 1 \mathrm{H}), 5.00-4.91(\mathrm{~m}, 2 \mathrm{H}), 2.21(\mathrm{~s}, 3 \mathrm{H}), 1.33-1.17(\mathrm{~m}, 12 \mathrm{H}) ;{ }^{13} \mathrm{C} \mathrm{NMR}$ $\left(\mathrm{CDCl}_{3}, 100 \mathrm{MHz}, \mathrm{ppm}\right):$ 169.3, 154.8, 151.1, 143.5, 132.2, 130.7, 118.7, 73.1, 71.1, 24.6, 21.9, 21.6; HRMS (ESI) calcd for $\mathrm{C}_{16} \mathrm{H}_{24} \mathrm{~N}_{3} \mathrm{O}_{7} \mathrm{~S}(\mathrm{M}+\mathrm{H})^{+} 402.1335$, found 402.1331 .

\section{Diisopropyl1-(naphthalen-2-ylsulfonyl)hydrazine-1,2-dicarboxylate (new compound)}

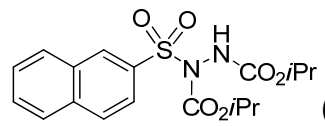

Compounds 31 were obtained in $60 \%$ yield according to the general procedure $(12 \mathrm{~h}) .{ }^{1} \mathrm{H}$ NMR (400 MHz, $\left.\mathrm{CDCl}_{3}\right) \delta 8.72(\mathrm{~s}, 1 \mathrm{H}), 8.12$ (d, $\left.J=8.6 \mathrm{~Hz}, 1 \mathrm{H}\right), 8.04-7.98(\mathrm{~m}, 2 \mathrm{H})$, 7.94 (d, $J=8.0 \mathrm{~Hz}, 1 \mathrm{H}), 7.71-7.62$ (m, 2H), 7.06 (brs, 1H), 5.05-4.99 (m, 1H), 4.94-4.88 $(\mathrm{m}, 1 \mathrm{H}), 1.37-1.28(\mathrm{~m}, 6 \mathrm{H}), 1.24-1.09(\mathrm{~m}, 6 \mathrm{H}) ;{ }^{13} \mathrm{C}$ NMR $\left(100 \mathrm{MHz}, \mathrm{CDCl}_{3}\right) \delta 154.5$, 151.0, 135.5, 135.0, 131.8, 129.6, 129.4, 128.8, 128.0, 127.5, 123.8, 73.0, 71.0, 21.9, 21.7, 21.6; HRMS (ESI) calcd for $\mathrm{C}_{18} \mathrm{H}_{22} \mathrm{~N}_{2} \mathrm{O}_{6} \mathrm{NaS}(\mathrm{M}+\mathrm{Na})^{+} 417.1096$, found 417.1099.

diisopropyl1-(trifluoromethylsulfonyl)hydrazine-1,2-dicarboxylate (new compound)

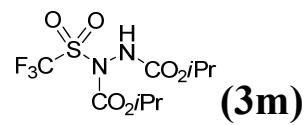

Compounds $\mathbf{3 m}$ were obtained in $87 \%$ yield according to the general procedure $(12 \mathrm{~h}) .{ }^{1} \mathrm{H}$ NMR (400 MHz, $\left.\mathrm{CDCl}_{3}\right) \delta 7.13$ (brs, $\left.1 \mathrm{H}\right), 5.18-5.11(\mathrm{~m}, 1 \mathrm{H}), 5.07-5.01(\mathrm{~m}, 1 \mathrm{H})$, 1.40-1.36 (m, 6H), $1.31(\mathrm{~d}, J=6.1 \mathrm{~Hz}, 6 \mathrm{H}) ;{ }^{13} \mathrm{C} \mathrm{NMR}\left(100 \mathrm{MHz}, \mathrm{CDCl}_{3}\right) \delta 154.1,149.6$, $130.9(\mathrm{~d}, J=3.6 \mathrm{~Hz}), 128.8(\mathrm{~d}, J=4.4 \mathrm{~Hz}), 119.3$ (q, $J=322.6 \mathrm{~Hz}), 75.5,71.9,21.7$, 21.4, 21.4; HRMS (ESI) calcd for $\mathrm{C}_{9} \mathrm{H}_{15} \mathrm{~N}_{2} \mathrm{O}_{6} \mathrm{~F}_{3} \mathrm{NaS}(\mathrm{M}+\mathrm{Na})^{+}$359.0501, found 359.0503 .

diethyl1-(phenylsulfonyl)hydrazine-1,2-dicarboxylate

Compound 3n was obtained in 95\% yield according to the general procedure $(12 \mathrm{~h}) .{ }^{1} \mathrm{H}$ $\operatorname{NMR}\left(400 \mathrm{MHz}, \mathrm{CDCl}_{3}\right) \delta 8.15(\mathrm{~d}, J=7.5 \mathrm{~Hz}, 2 \mathrm{H}), 7.67$ (t, $\left.J=7.4 \mathrm{~Hz}, 1 \mathrm{H}\right), 7.56$ (t, $J=$ $8.1 \mathrm{~Hz}, 2 \mathrm{H}), 7.15$ (brs, 1H), 4.28-4.20 (m, 4H), 1.34 (t, $J=6.6 \mathrm{~Hz}, 3 \mathrm{H}), 1.20$ (t, $J=6.9$ 
$\mathrm{Hz}, 3 \mathrm{H}) ;{ }^{13} \mathrm{C}$ NMR $\left(100 \mathrm{MHz}, \mathrm{CDCl}_{3}\right) \delta 154.6,150.7,137.2,131.9,131.1,129.5,73.2$, 71.1, 21.9, 21.6, 21.6; HRMS (ESI) calcd for $\mathrm{C}_{12} \mathrm{H}_{16} \mathrm{~N}_{2} \mathrm{O}_{6} \mathrm{NaS}(\mathrm{M}+\mathrm{Na})^{+} 339.0627$, found 339.0627 .

diethyl1-(4-fluorophenylsulfonyl)hydrazine-1,2-dicarboxylate (new compound)

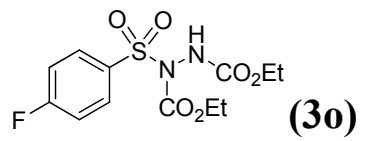

Compounds 30 were obtained in $76 \%$ yield according to the general procedure $(12 \mathrm{~h}) .{ }^{1} \mathrm{H}$ NMR $\left(400 \mathrm{MHz}, \mathrm{CDCl}_{3}\right) \delta 8.16\left(\mathrm{dd}, J_{l}=8.5 \mathrm{~Hz}, J_{2}=4.6 \mathrm{~Hz}, 2 \mathrm{H}\right), 7.36(\mathrm{brs}, 1 \mathrm{H}), 7.22(\mathrm{t}$, $J=8.5 \mathrm{~Hz}, 2 \mathrm{H}), 4.27-4.20(\mathrm{~m}, 4 \mathrm{H}), 1.32(\mathrm{t}, J=6.8 \mathrm{~Hz}, 3 \mathrm{H}), 1.21(\mathrm{t}, J=6.9 \mathrm{~Hz}, 3 \mathrm{H}) ;{ }^{13} \mathrm{C}$ NMR $\left(100 \mathrm{MHz}, \mathrm{CDCl}_{3}\right) \delta 166.1(\mathrm{~d}, J=255.6 \mathrm{~Hz}), 155.0,151.3,133.8(\mathrm{~d}, J=2.6 \mathrm{~Hz})$, $132.6(\mathrm{~d}, J=9.7 \mathrm{~Hz}), 115.9$ (d, $J=22.8 \mathrm{~Hz}), 64.5,63.0,14.3$, 14.0; HRMS (ESI) calcd for $\mathrm{C}_{12} \mathrm{H}_{15} \mathrm{~N}_{2} \mathrm{O}_{6} \mathrm{FNaS}(\mathrm{M}+\mathrm{Na})^{+}$357.0533, found 357.0533.

\section{diethyl 1-(2-bromophenylsulfonyl)hydrazine-1,2-dicarboxylate (new compound)}

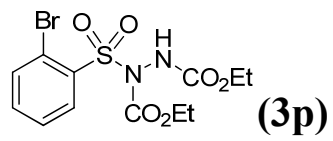

Compound $3 \mathbf{p}$ was obtained in $73 \%$ yield according to the general procedure $(12 \mathrm{~h}) .{ }^{1} \mathrm{H}$ NMR $\left(400 \mathrm{MHz}, \mathrm{CDCl}_{3}\right) \delta 8.36(\mathrm{~d}, J=6.8 \mathrm{~Hz}, 1 \mathrm{H}), 7.76\left(\mathrm{dd}, J_{1}=1.2 \mathrm{~Hz}, J_{2}=7.8 \mathrm{~Hz}\right.$, 1H), 7.58-7.48 (m, 2H), 7.13(brs, 1H), 4.35-4.29 (m, 2H), 4.21-4.15 (m, 2H), 1.35 (t, J= $7.1 \mathrm{~Hz}, 3 \mathrm{H}), 1.15(\mathrm{~d}, J=7.0 \mathrm{~Hz}, 3 \mathrm{H}) ;{ }^{13} \mathrm{C} \mathrm{NMR}\left(100 \mathrm{MHz}, \mathrm{CDCl}_{3}\right) \delta 155.5,151.1,138.0$, 135.2, 134.8, 133.8, 127.8, 120.0, 64.5, 63.2, 14.3, 13.9; $\left.\mathrm{MS}_{(\mathrm{ESI}}^{+}\right):[\mathrm{M}+\mathrm{H}]^{+} 395.0$; HRMS (ESI) calcd for $\mathrm{C}_{12} \mathrm{H}_{15} \mathrm{~N}_{2} \mathrm{O}_{6} \mathrm{BrNaS}(\mathrm{M}+\mathrm{Na})^{+} 416.9732$, found 416.9734 .

\section{di-tert-butyl1-(phenylsulfonyl)hydrazine-1,2-dicarboxylate (new compound)}

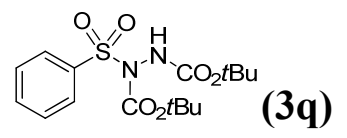

Compounds $\mathbf{3 q}$ were obtained in $98 \%$ yield according to the general procedure $(12 \mathrm{~h}) .{ }^{1} \mathrm{H}$ NMR (400 MHz, $\left.\mathrm{CDCl}_{3}\right) \delta 8.15(\mathrm{~d}, J=7.5 \mathrm{~Hz}, 2 \mathrm{H}), 7.65$ (t, $\left.J=7.1 \mathrm{~Hz}, 1 \mathrm{H}\right), 7.54$ (t, $J=$ $7.9 \mathrm{~Hz}, 2 \mathrm{H}), 6.94$ (brs, 1H), $1.51(\mathrm{~s}, 9 \mathrm{H}), 1.38(\mathrm{~s}, 9 \mathrm{H}) ;{ }^{13} \mathrm{C} \mathrm{NMR}\left(100 \mathrm{MHz}, \mathrm{CDCl}_{3}\right) \delta$ 153.6, 149.8, 138.7, 133.8, 129.0, 128.6, 85.5, 82.5, 28.1, 27.8; HRMS (ESI) calcd for $\mathrm{C}_{16} \mathrm{H}_{24} \mathrm{~N}_{2} \mathrm{O}_{6} \mathrm{NaS}(\mathrm{M}+\mathrm{Na})^{+}$395.1253, found 395.1258. 
di-tert-butyl 1-tosylhydrazine-1,2-dicarboxylate (new compound)

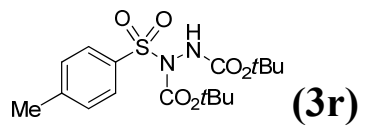

Compounds $3 \mathbf{r}$ were obtained in $84 \%$ yield according to the general procedure $(12 \mathrm{~h}) .{ }^{1} \mathrm{H}$ NMR (400 MHz, $\left.\mathrm{CDCl}_{3}\right) \delta 8.00(\mathrm{~d}, J=8.0 \mathrm{~Hz}, 2 \mathrm{H}), 7.33(\mathrm{~d}, J=8.1 \mathrm{~Hz}, 1 \mathrm{H}), 6.89$ (brs, $1 \mathrm{H}), 2.46(\mathrm{~s}, 3 \mathrm{H}), 1.51(\mathrm{~s}, 9 \mathrm{H}), 1.40(\mathrm{~s}, 9 \mathrm{H}) ;{ }^{13} \mathrm{C} \mathrm{NMR}\left(100 \mathrm{MHz}, \mathrm{CDCl}_{3}\right) \delta 153.6,149.9$, 144.9, 135.7, 129.2, 129.1, 85.3, 82.4, 28.1, 27.8, 21.7; HRMS (ESI) calcd for $\mathrm{C}_{17} \mathrm{H}_{26} \mathrm{~N}_{2} \mathrm{O}_{6} \mathrm{NaS}(\mathrm{M}+\mathrm{Na})^{+} 409.1409$, found 409.1410.

di-tert-butyl1-(4-chlorophenylsulfonyl)hydrazine-1,2-dicarboxylate(new compound)

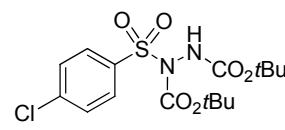

(3s)

Compounds $3 \mathrm{~s}$ were obtained in $84 \%$ yield according to the general procedure $(12 \mathrm{~h}) .{ }^{1} \mathrm{H}$ NMR (400 MHz, $\left.\mathrm{CDCl}_{3}\right) \delta 8.08(\mathrm{~d}, J=8.5 \mathrm{~Hz}, 2 \mathrm{H}), 7.51(\mathrm{~d}, J=8.6 \mathrm{~Hz}, 2 \mathrm{H}), 6.96$ (brs, 1H), $1.50(\mathrm{~s}, 9 \mathrm{H}), 1.40(\mathrm{~s}, 9 \mathrm{H}) ;{ }^{13} \mathrm{C} \mathrm{NMR}\left(100 \mathrm{MHz}, \mathrm{CDCl}_{3}\right) \delta 153.6,149.7,140.5,137.1$,

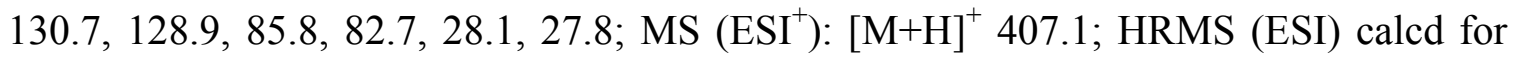
$\mathrm{C}_{16} \mathrm{H}_{23} \mathrm{~N}_{2} \mathrm{O}_{6} \mathrm{ClNaS}(\mathrm{M}+\mathrm{Na})^{+} 429.0863$, found 429.0862 .

di-tert-butyl1-(4-fluorophenylsulfonyl)hydrazine-1,2-dicarboxylate (new compound)

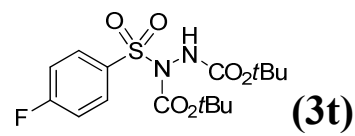

Compounds $3 \mathbf{t}$ were obtained in $83 \%$ yield according to the general procedure $(12 \mathrm{~h}) .{ }^{1} \mathrm{H}$ NMR (400 MHz, $\left.\mathrm{CDCl}_{3}\right) \delta 8.17\left(\mathrm{dd}, J_{1}=8.4, J_{2}=8.0 \mathrm{~Hz}, 2 \mathrm{H}\right), 7.22(\mathrm{~d}, J=8.4 \mathrm{~Hz}, 2 \mathrm{H})$, 6.92 (brs, 1H), 1.51 (s, 9H), $1.40(\mathrm{~s}, 9 \mathrm{H}) ;{ }^{13} \mathrm{C} \mathrm{NMR}\left(100 \mathrm{MHz}, \mathrm{CDCl}_{3}\right) \delta 165.9(\mathrm{~d}, J=$ $255.0 \mathrm{~Hz}), 153.6,149.7,134.5$ (d, $J=34.0 \mathrm{~Hz}), 132.1$ (d, $J=9.4 \mathrm{~Hz}), 115.8$ (d, $J=22.7$ $\mathrm{Hz}), 85.7,82.6,28.1,27.8$; HRMS (ESI) calcd for $\mathrm{C}_{16} \mathrm{H}_{23} \mathrm{~N}_{2} \mathrm{O}_{6} \mathrm{FNaS}(\mathrm{M}+\mathrm{Na})^{+}$413.1159, found 413.1155 .

dibenzyl1-tosylhydrazine-1,2-dicarboxylate (new compound)

Compounds $3 \mathbf{u}$ were obtained in $80 \%$ yield according to the general procedure $(12 \mathrm{~h}) .{ }^{1} \mathrm{H}$ NMR (400 MHz, $\left.\mathrm{CDCl}_{3}\right) \delta 7.92(\mathrm{~d}, J=8.0 \mathrm{~Hz}, 2 \mathrm{H}), 7.41$ (brs, $\left.1 \mathrm{H}\right), 7.37-7.22(\mathrm{~m}, 12 \mathrm{H})$, 5.26-5.04 (m, 4H), $2.43(\mathrm{~s}, 3 \mathrm{H}) ;{ }^{13} \mathrm{C} \mathrm{NMR}\left(100 \mathrm{MHz}, \mathrm{CDCl}_{3}\right) \delta 154.9,151.4,145.4$, 
135.3, 134.7, 134.2, 129.6, 129.3, 128.7, 128.6, 128.6, 128.5, 128.4, 128.1, 69.7, 68.3, 21.7; HRMS (ESI) calcd for $\mathrm{C}_{23} \mathrm{H}_{22} \mathrm{~N}_{2} \mathrm{O}_{6} \mathrm{NaS}(\mathrm{M}+\mathrm{Na})^{+} 477.1096$, found 477.1101.

dibenzyl1-(4-chlorophenylsulfonyl)hydrazine-1,2-dicarboxylate (new compound)

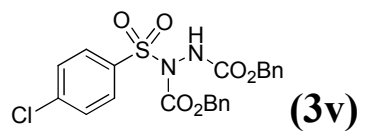

Compounds $3 \mathbf{v}$ were obtained in $83 \%$ yield according to the general procedure $(12 \mathrm{~h}) .{ }^{1} \mathrm{H}$ NMR (400 MHz, $\left.\mathrm{CDCl}_{3}\right) \delta 7.95$ (d, $\left.J=8.5 \mathrm{~Hz}, 2 \mathrm{H}\right), 7.55$ (brs, 1H), 7.37-7.28 (m, 10H), 7.21 (d, $J=7.6 \mathrm{~Hz}, 2 \mathrm{H}), 5.22-5.08(\mathrm{~m}, 4 \mathrm{H}) ;{ }^{13} \mathrm{C} \mathrm{NMR}\left(100 \mathrm{MHz}, \mathrm{CDCl}_{3}\right) \delta 154.9,151.2$, 140.9, 136.1, 135.2, 134.0, 131.1, 130.7, 128.9, 128.9, 128.7, 128.6, 128.5, 128.2, 70.0,

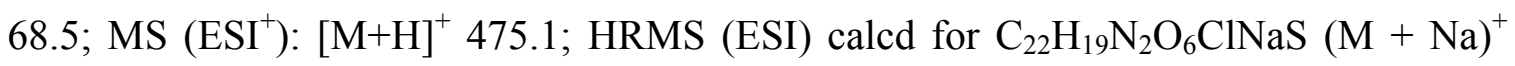
497.0550, found 497.0552.

dibenzyl1-(4-bromophenylsulfonyl)hydrazine-1,2-dicarboxylate (new compound)

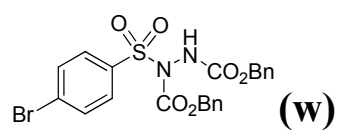

Compounds $3 \mathbf{w}$ were obtained in $76 \%$ yield according to the general procedure (12h). ${ }^{1} \mathrm{H}$ NMR (400 MHz, $\left.\mathrm{CDCl}_{3}\right) \delta 7.87(\mathrm{~d}, J=8.4 \mathrm{~Hz}, 2 \mathrm{H}), 7.54(\mathrm{~d}, J=8.4 \mathrm{~Hz}, 2 \mathrm{H}), 7.44$ (brs $1 \mathrm{H}), 7.37-7.28(\mathrm{~m}, 8 \mathrm{H}), 7.22(\mathrm{~d}, J=6.3 \mathrm{~Hz}, 2 \mathrm{H}), 5.22-5.08(\mathrm{~m}, 4 \mathrm{H}) ;{ }^{13} \mathrm{C} \mathrm{NMR}(100 \mathrm{MHz}$, $\left.\mathrm{CDCl}_{3}\right) \delta 154.9,151.1,136.5,135.1,133.9,131.9,131.1,130.7,130.0,128.9,128.7$, 128.6, 128.5, 128.2, 70.1, 68.6; $\mathrm{MS}\left(\mathrm{ESI}^{+}\right):[\mathrm{M}+\mathrm{H}]^{+}$519.0; HRMS (ESI) calcd for $\mathrm{C}_{22} \mathrm{H}_{19} \mathrm{~N}_{2} \mathrm{O}_{6} \mathrm{BrNaS}(\mathrm{M}+\mathrm{Na})^{+} 541.0045$, found 541.0048 .

\section{Copies of NMR Spectra for $3 a-3 w$}




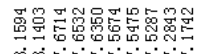

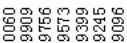

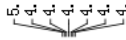

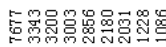

舟

广过计士
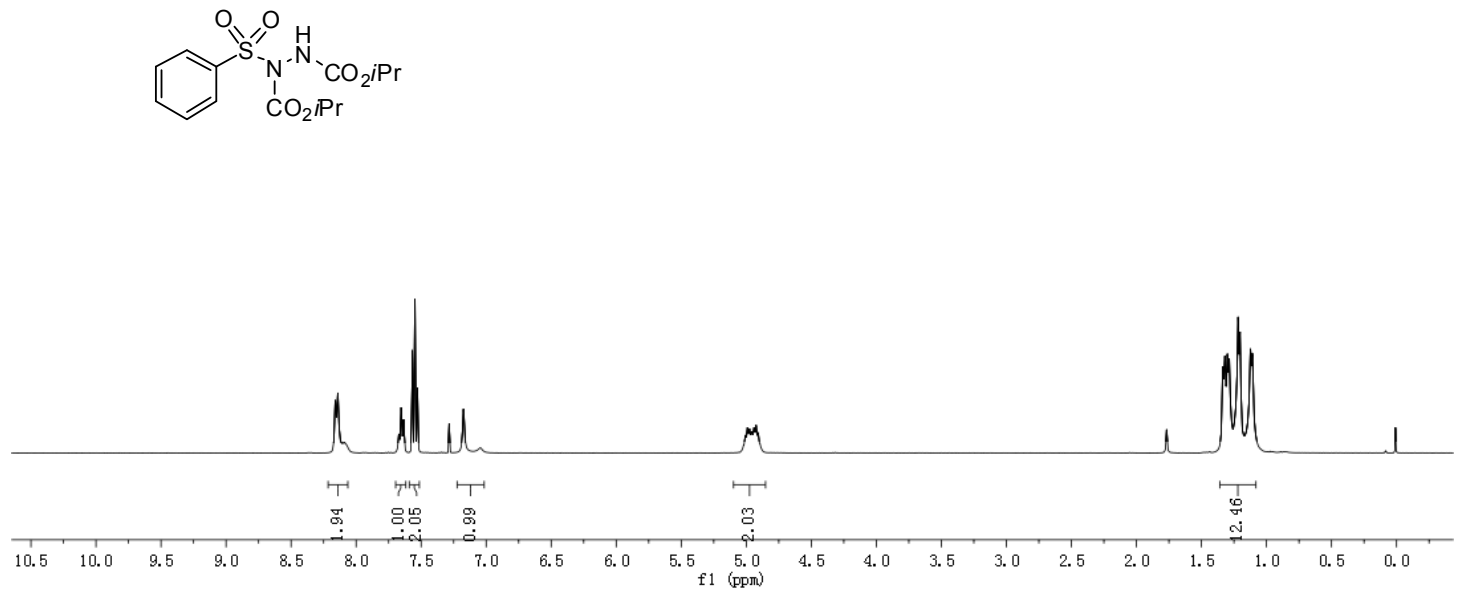

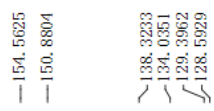

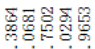

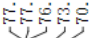

옳욤

$\overline{\mathrm{V}}$

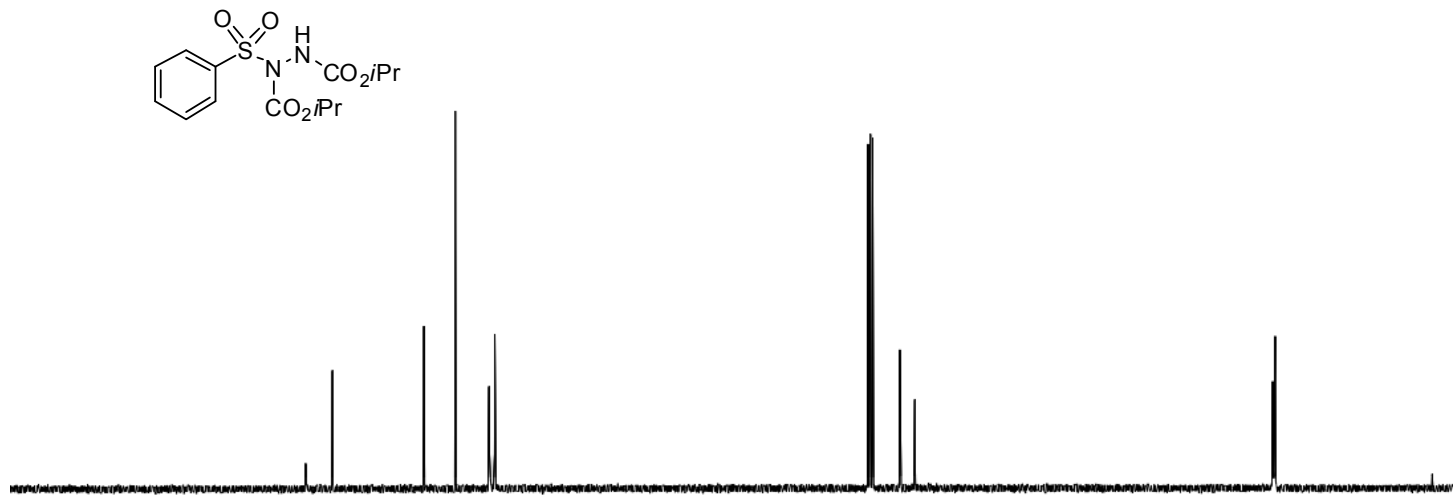

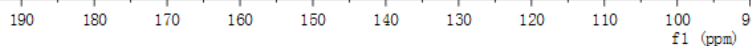



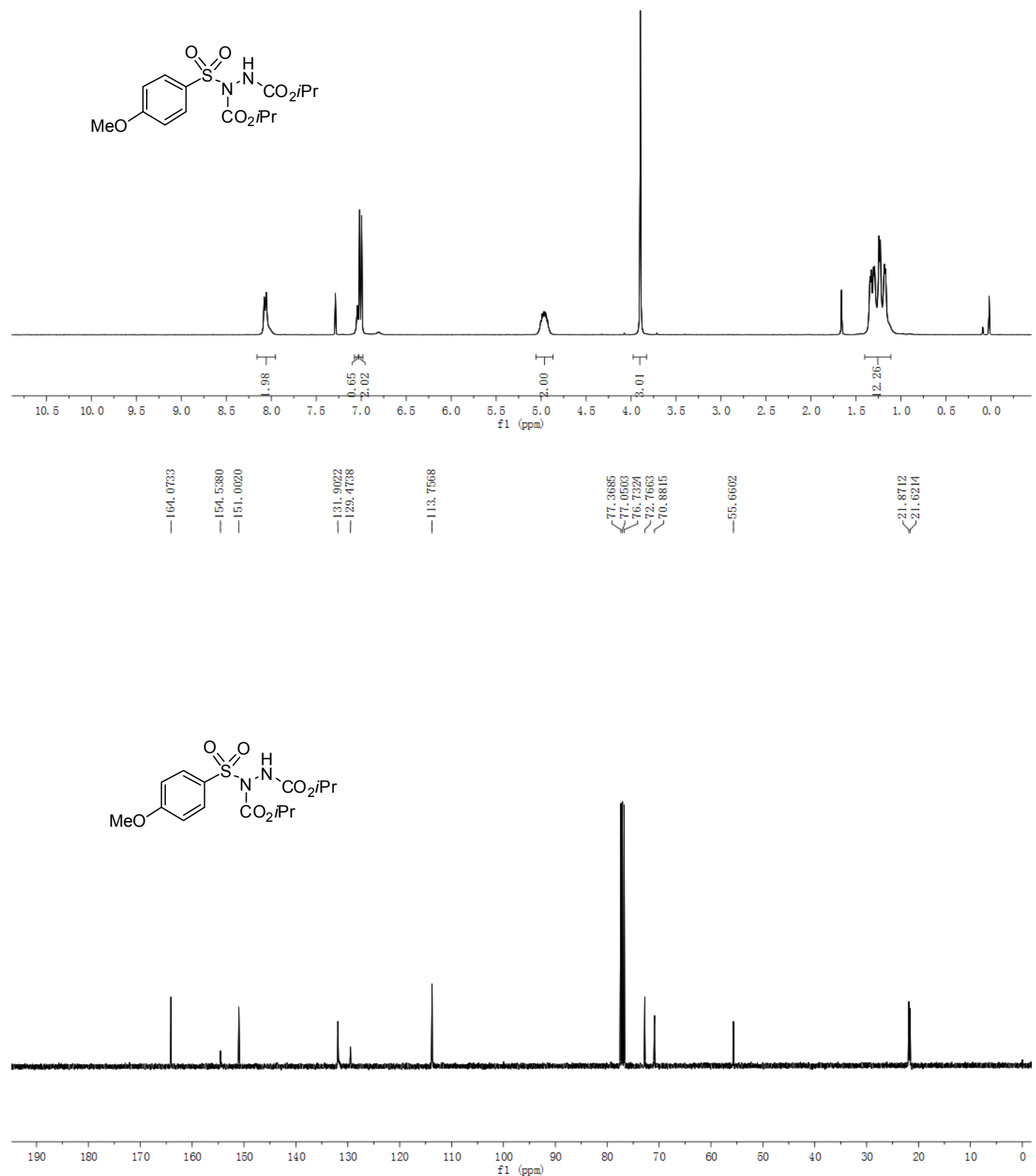

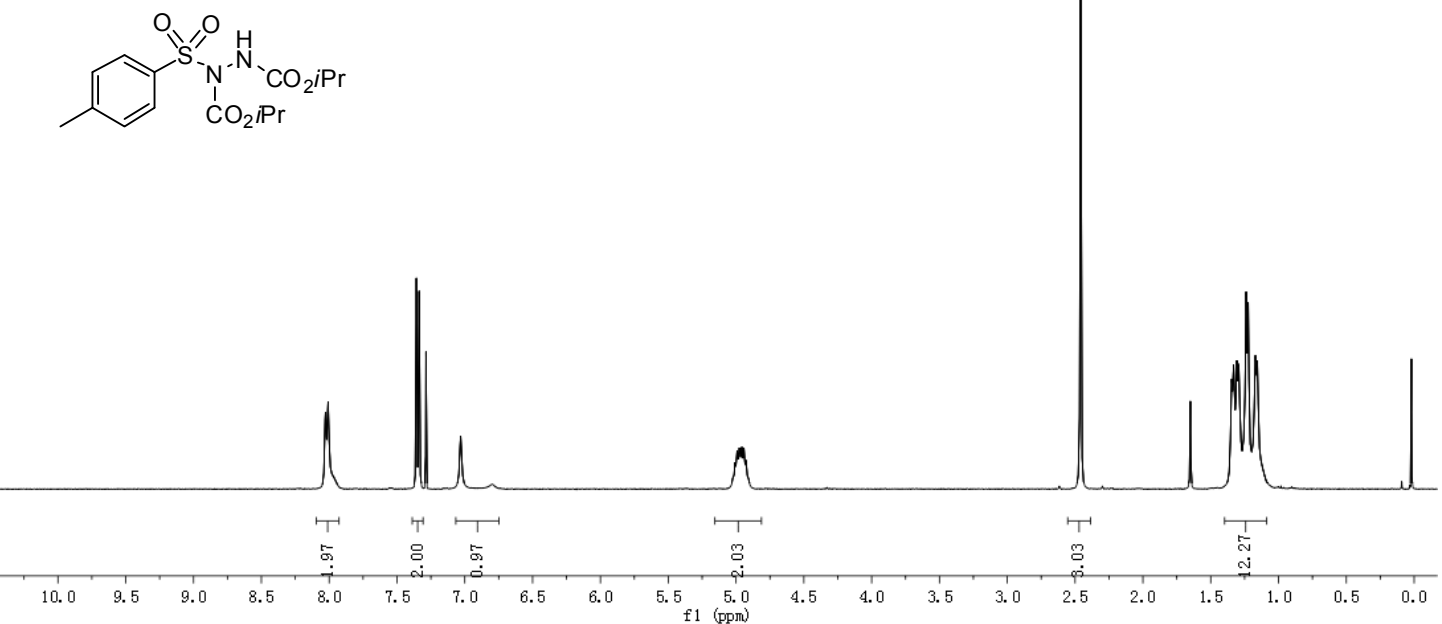

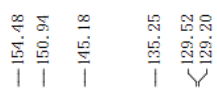

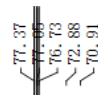

$\infty \%$
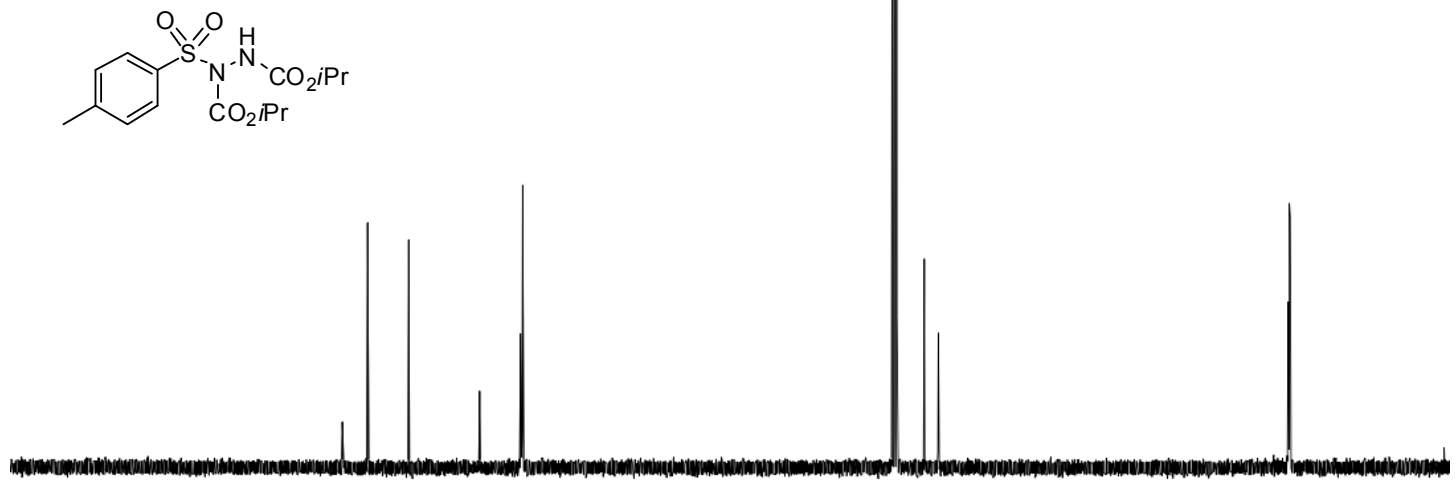

$\stackrel{7}{200}$
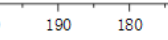

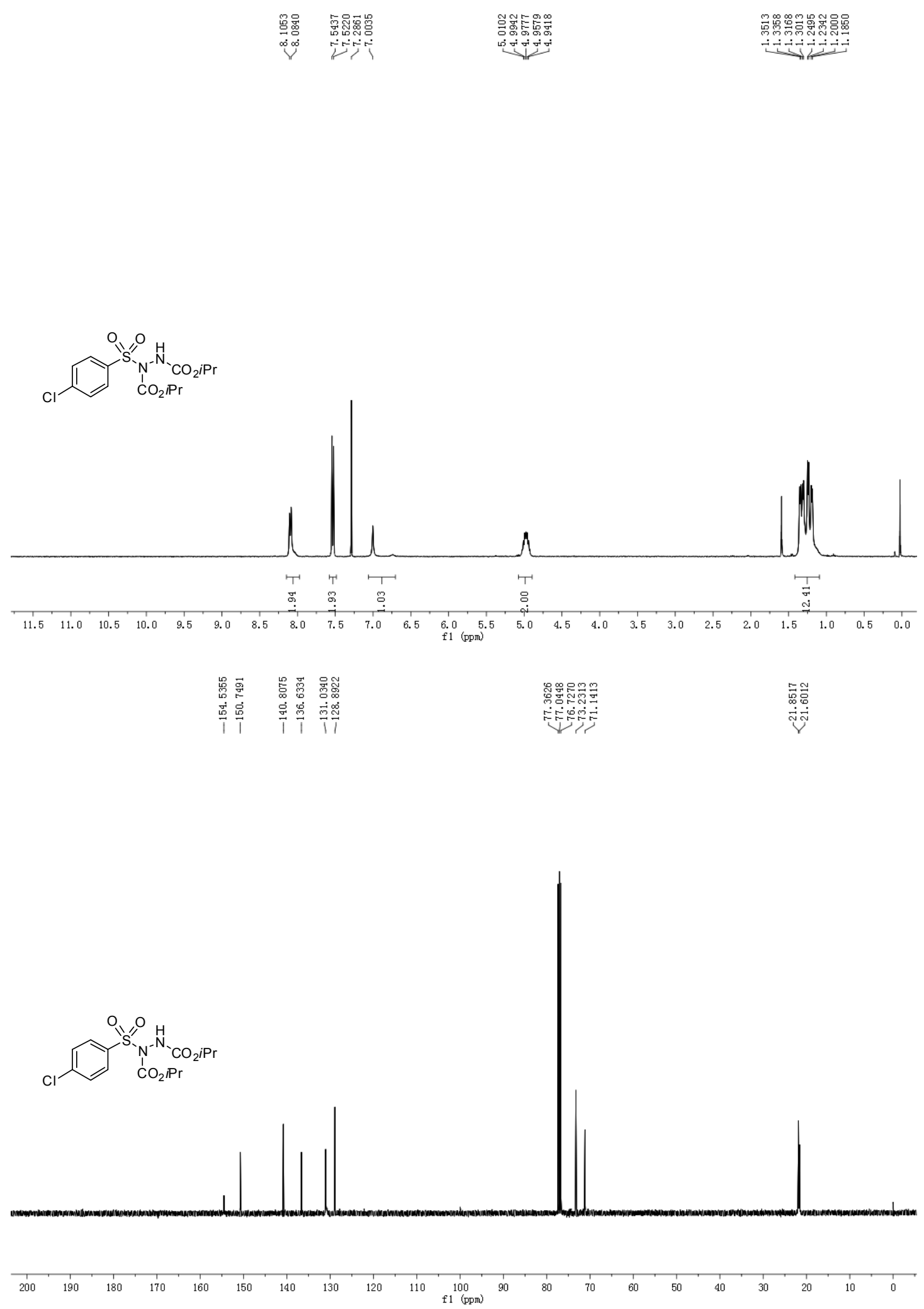

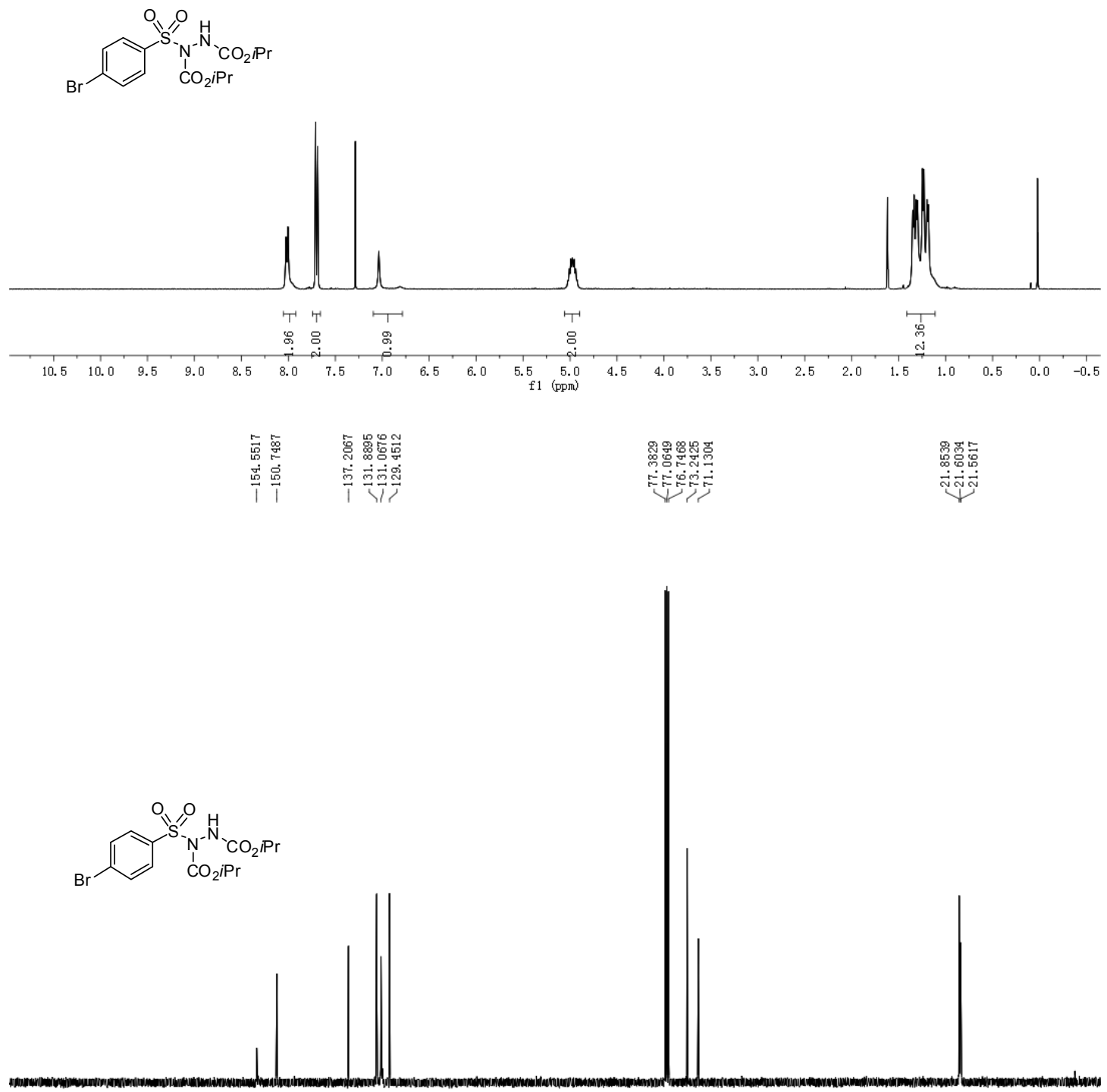

200
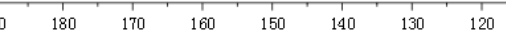

10090 

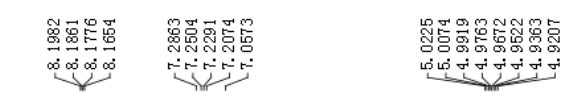

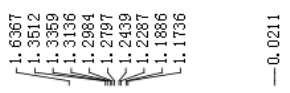
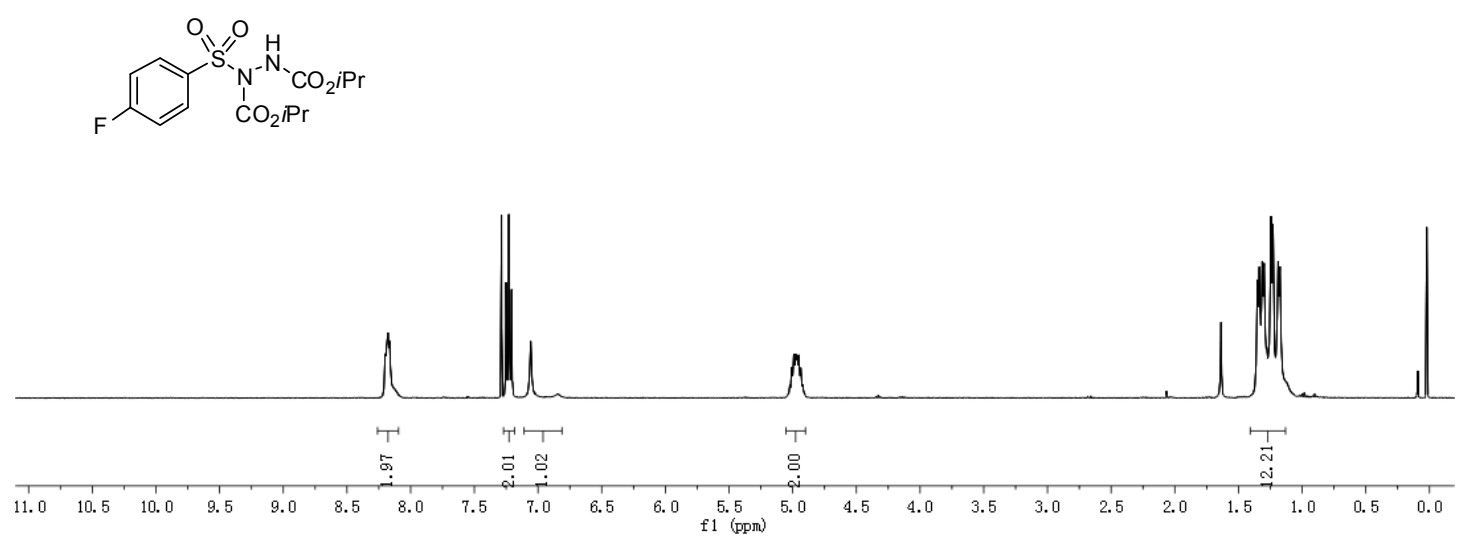

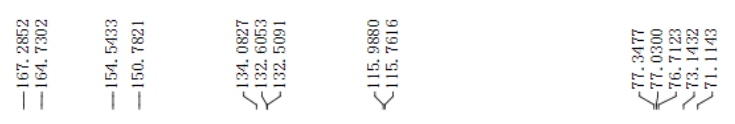

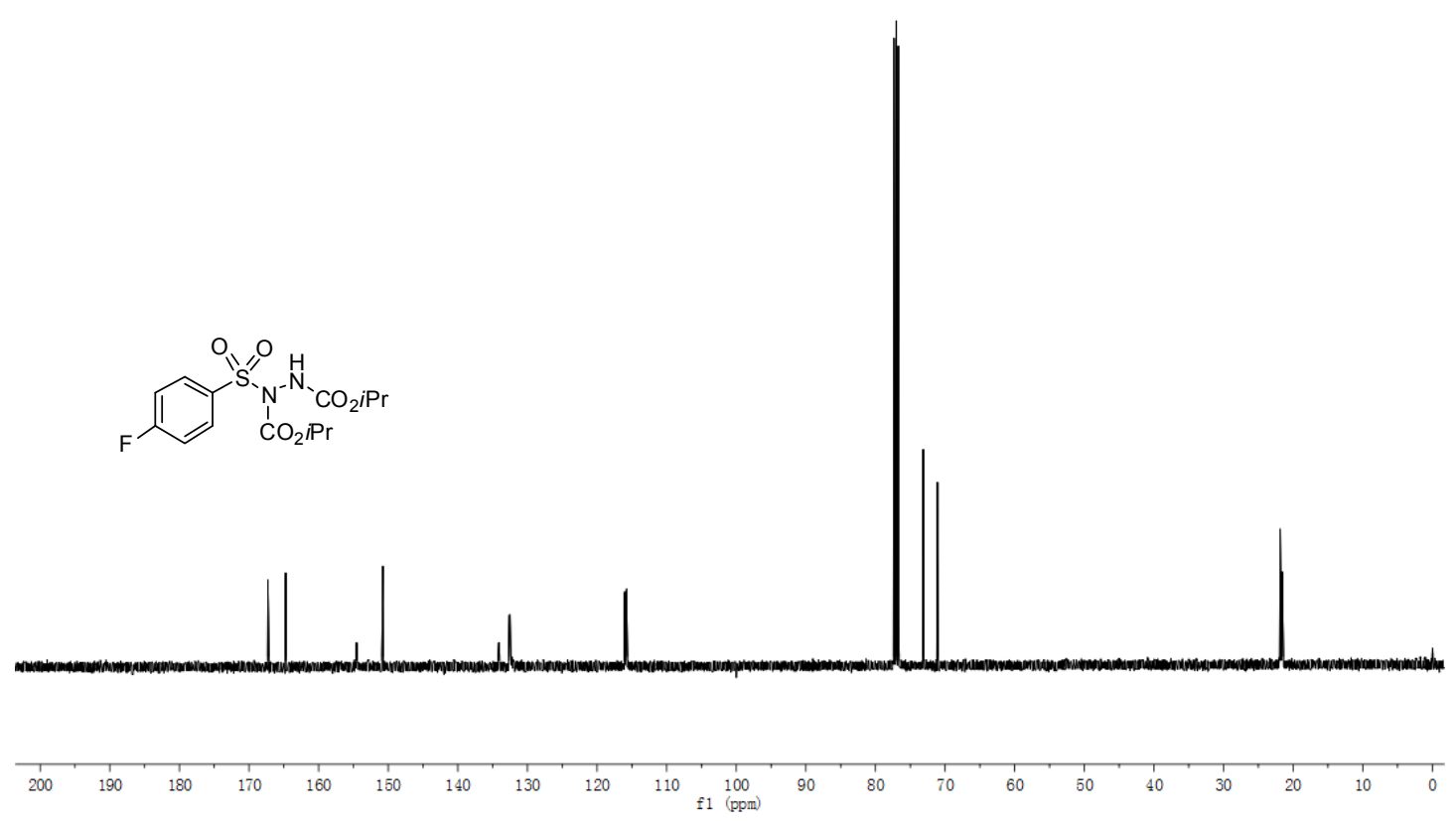




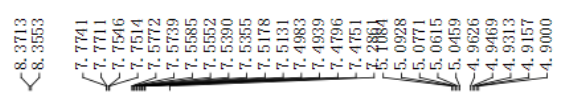

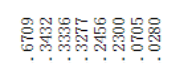

讨讨于
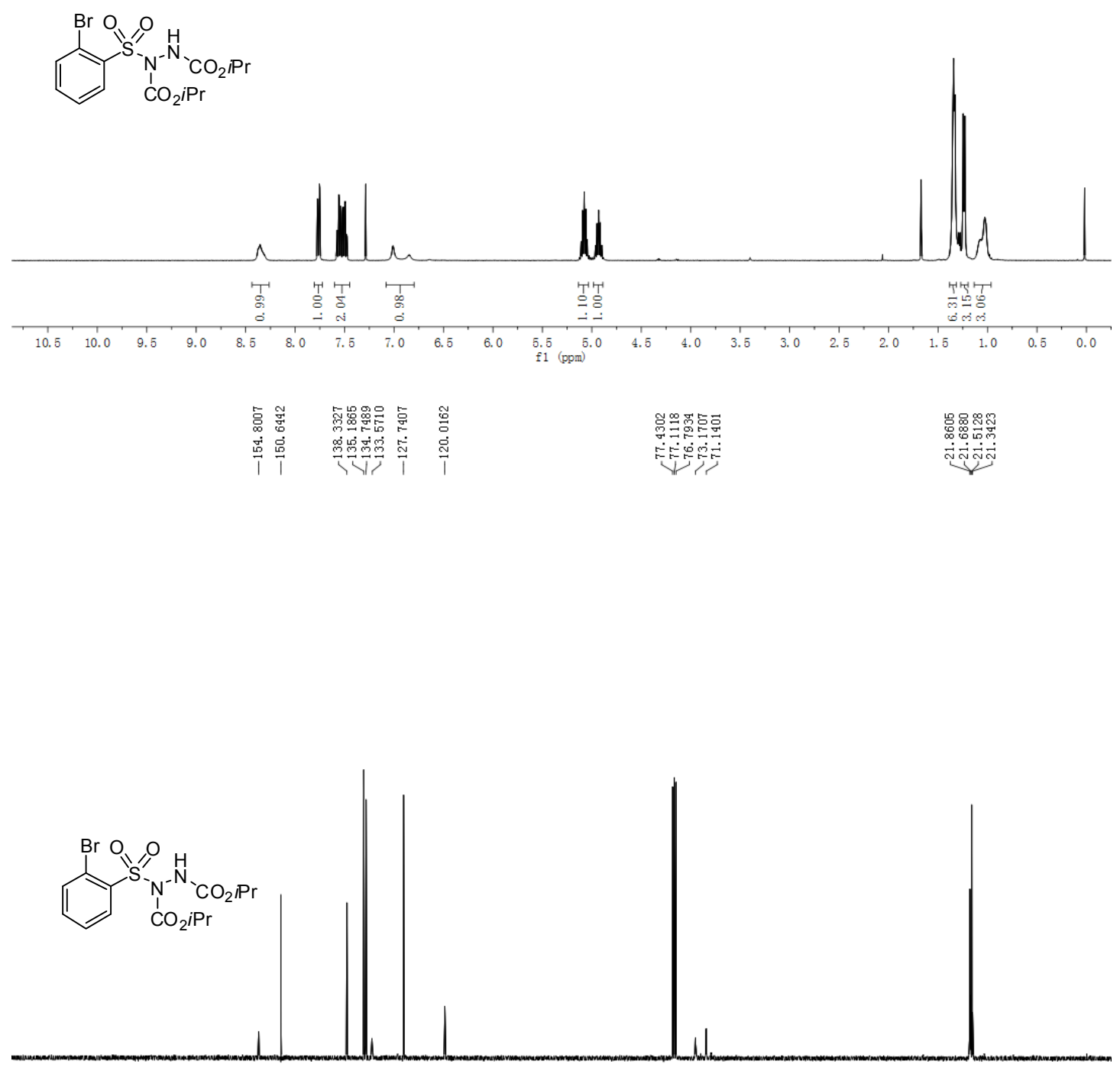

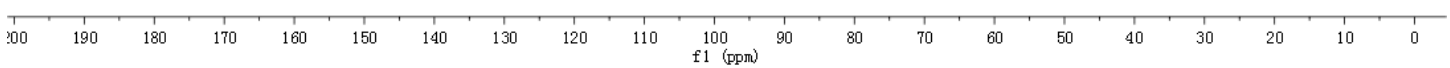




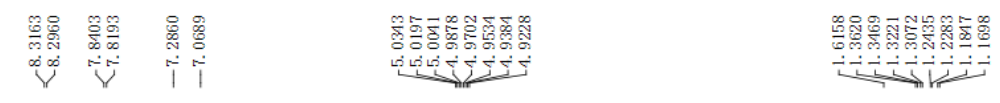

$\overbrace{\mathrm{CO}_{2} \mathrm{Pr}}^{\mathrm{O} \mathrm{Pr}}$

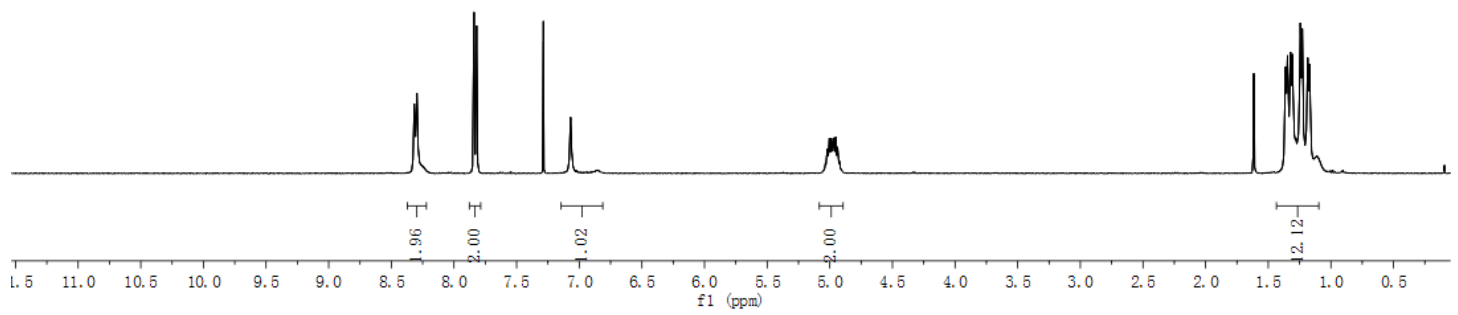

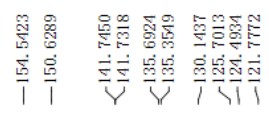

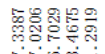

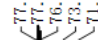

象

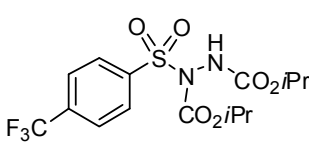
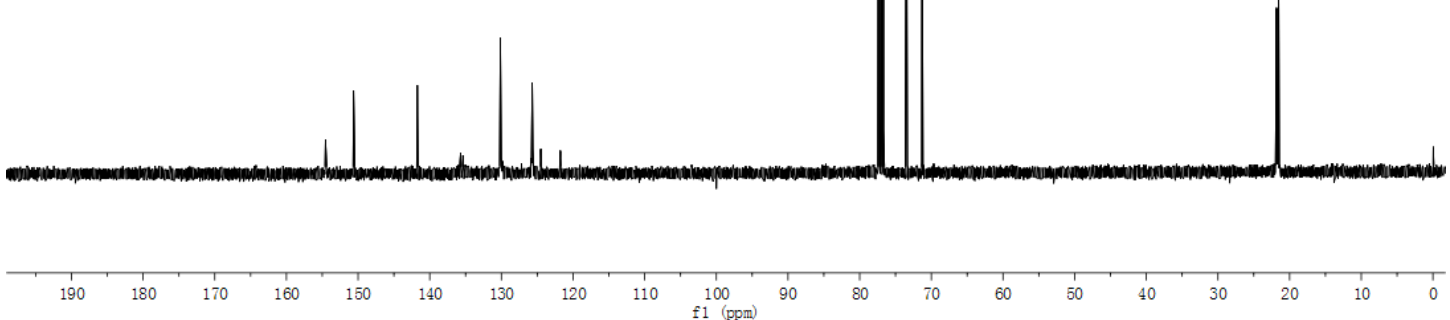

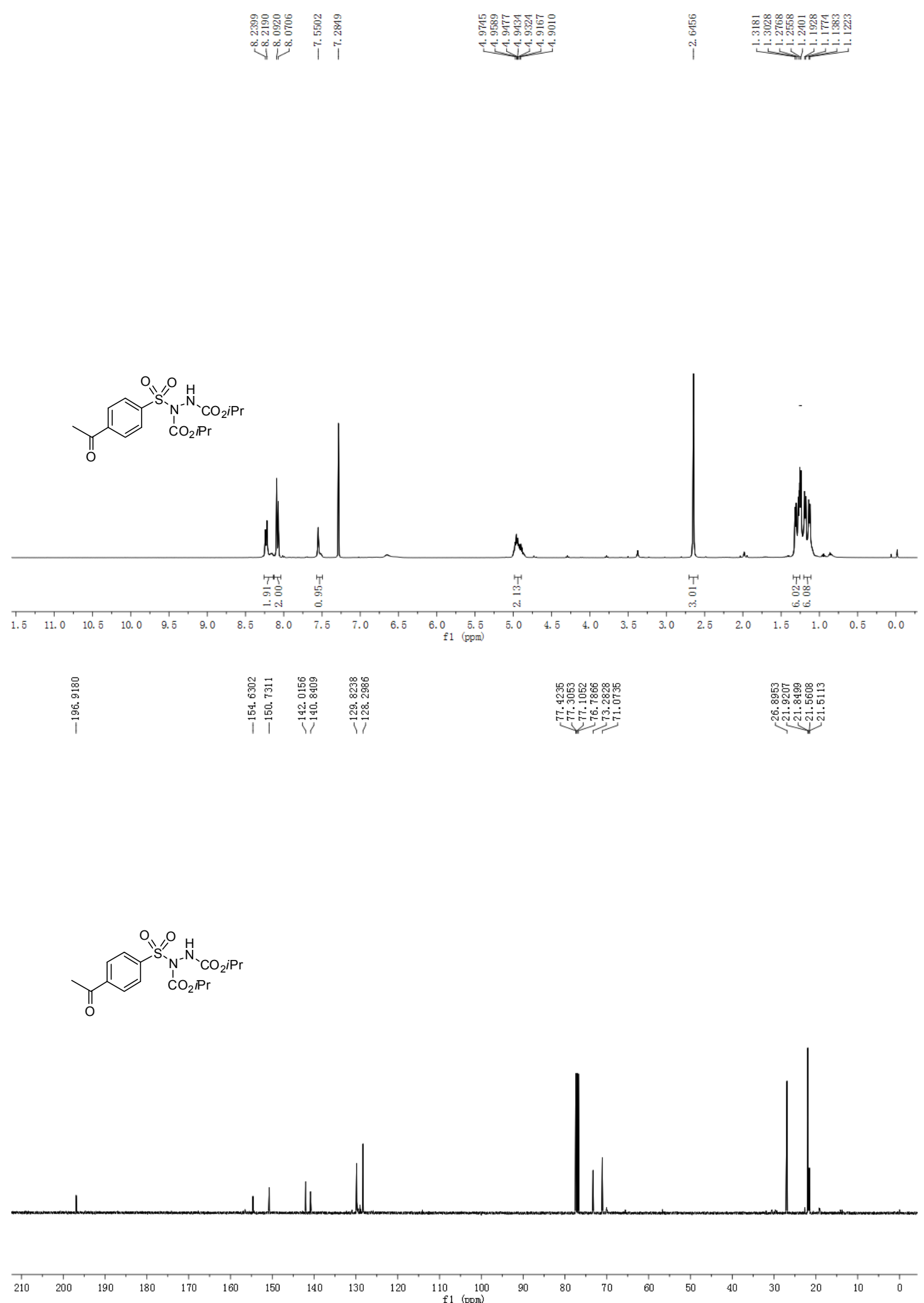


\begin{tabular}{|c|c|}
\hline & 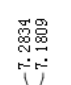 \\
\hline
\end{tabular}
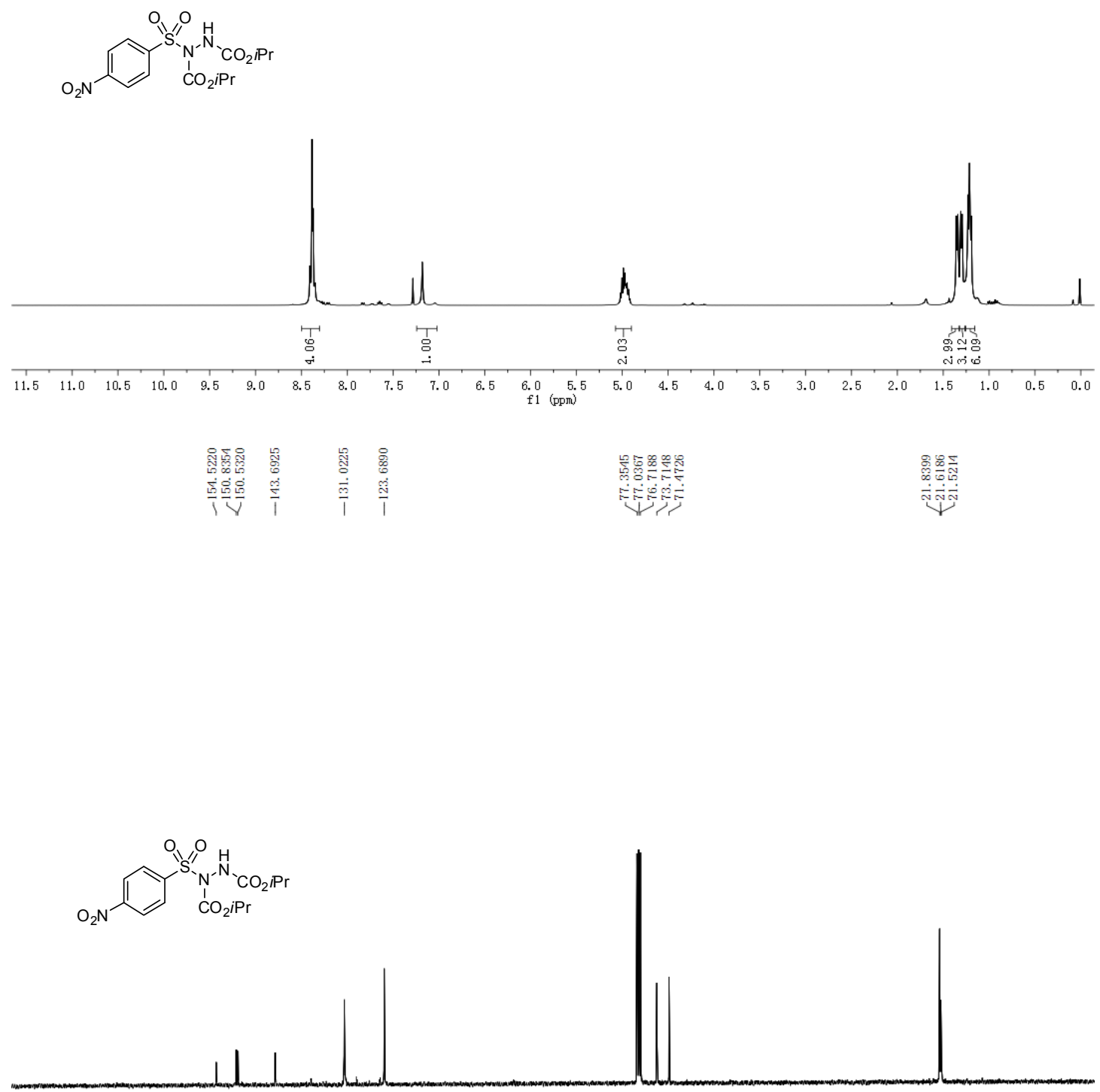

$\underset{190}{\top}$
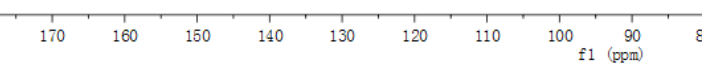


\begin{tabular}{|c|c|}
\hline 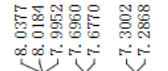 & 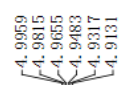 \\
\hline
\end{tabular}<smiles>CC(=O)Nc1ccc(S(=O)(=O)N(NOC(C)=O)C(=O)OC(C)C)cc1</smiles>

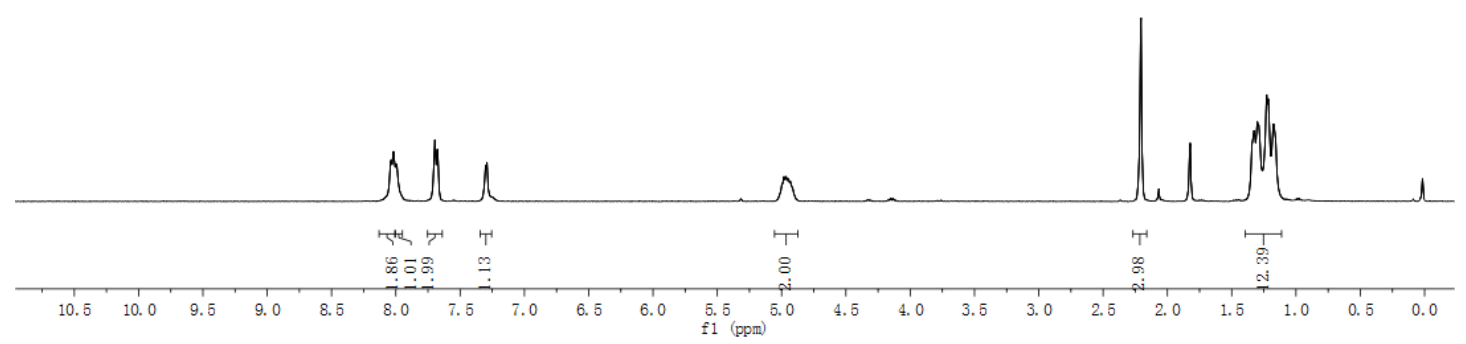<smiles>CCOC(=O)N(NOC(C)=O)S(=O)(=O)c1ccc(NC(C)=O)cc1</smiles>
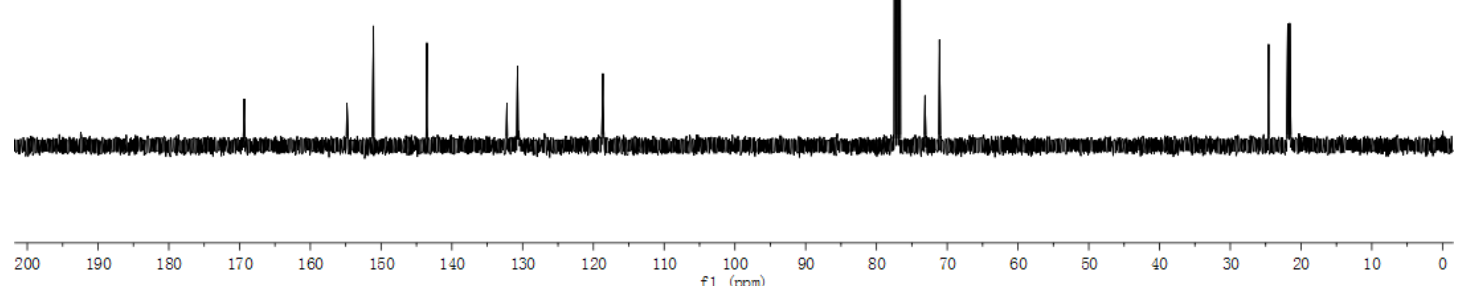


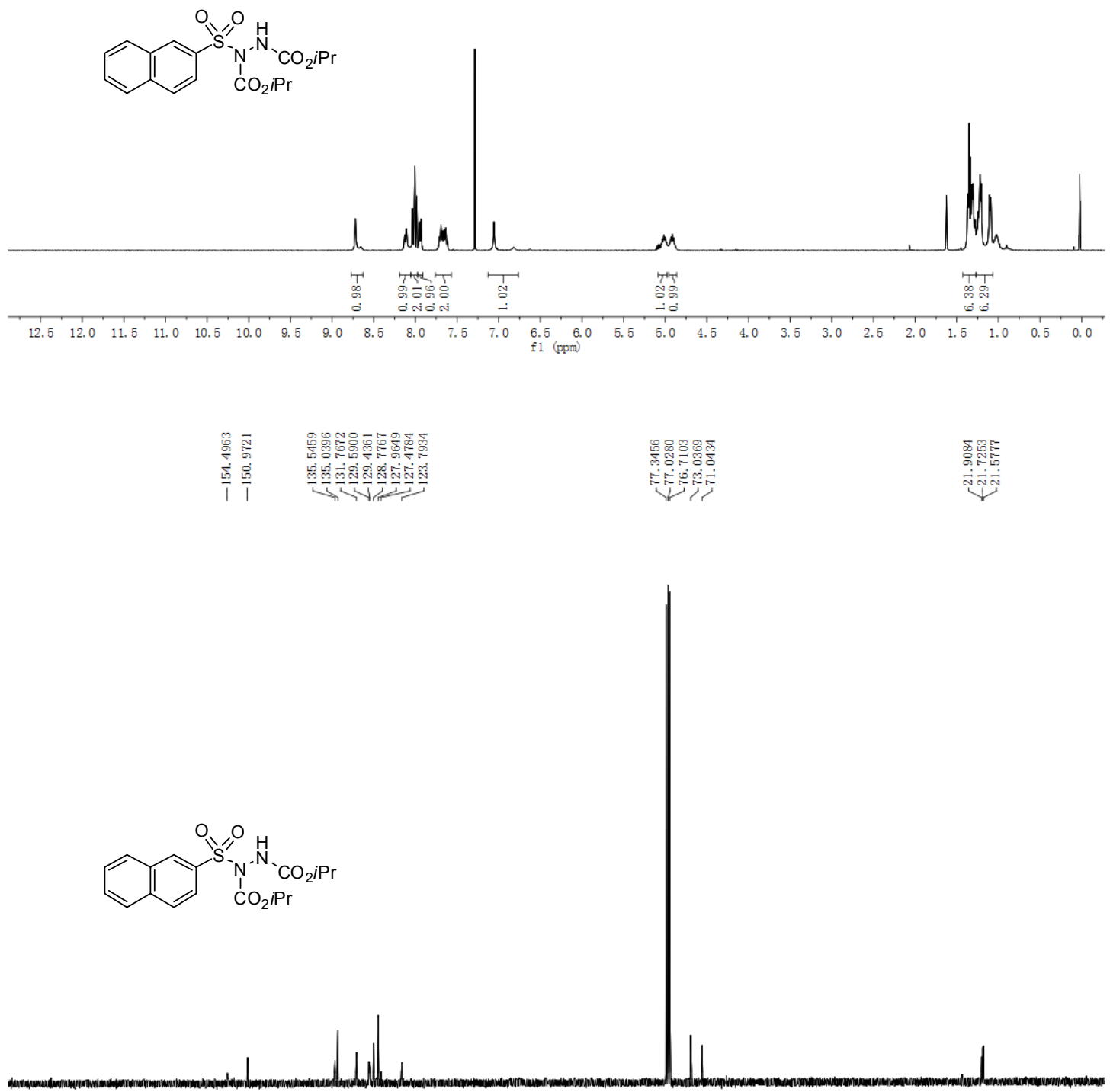

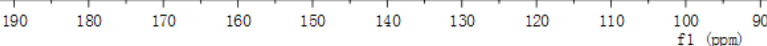




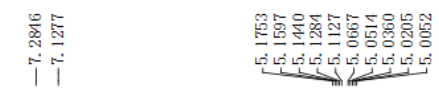

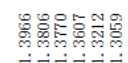

证立
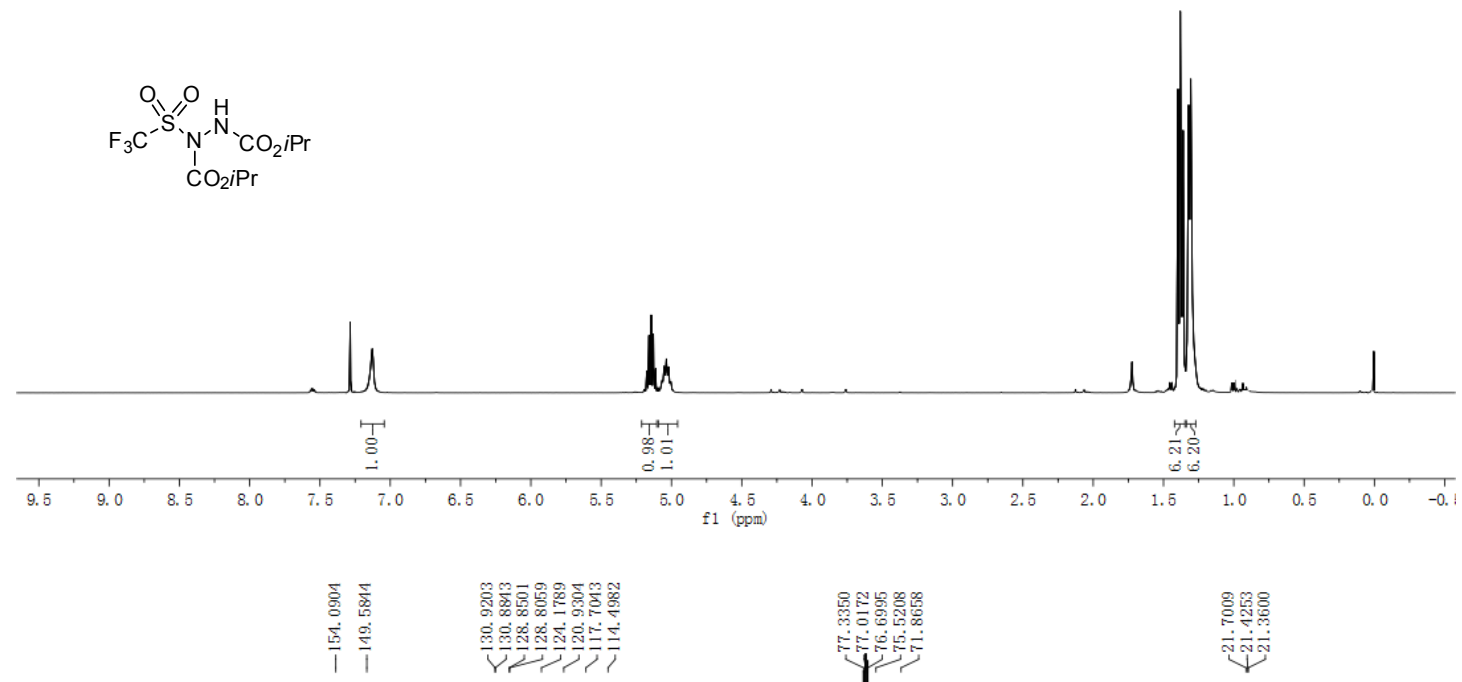

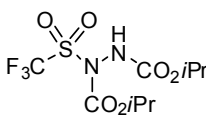
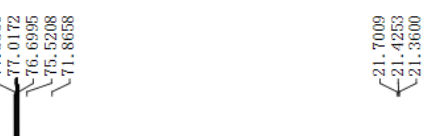

نิ
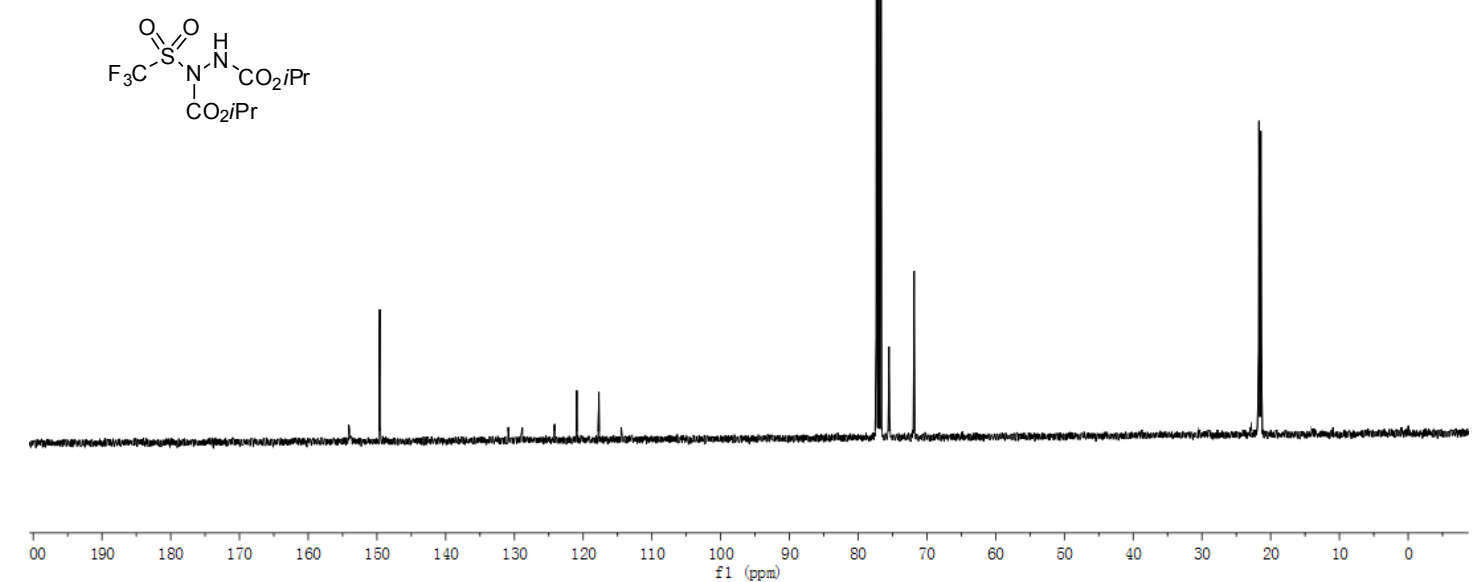


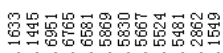

$y$
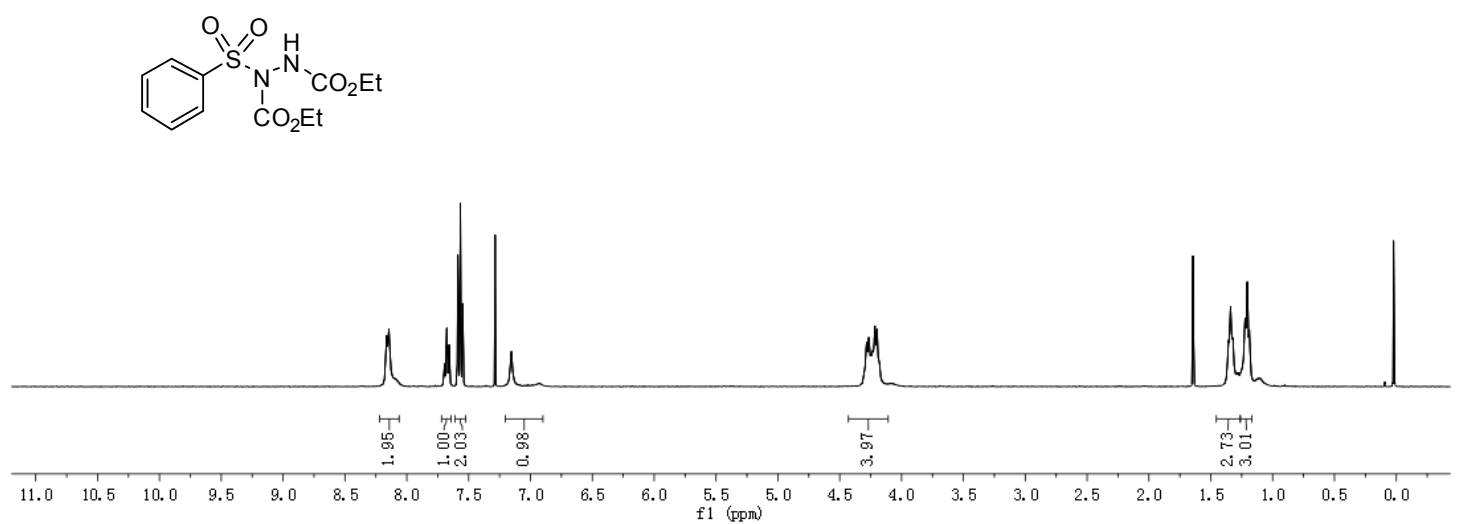

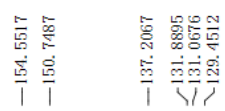

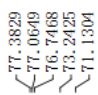
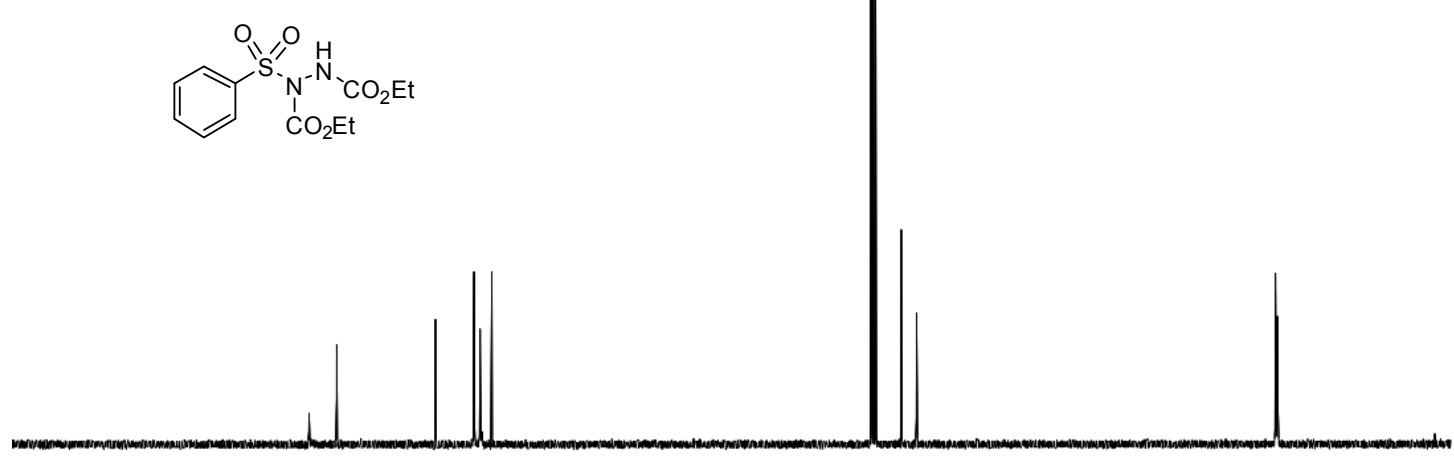

190
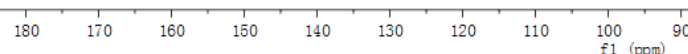


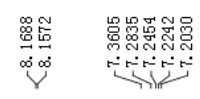

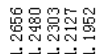

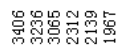
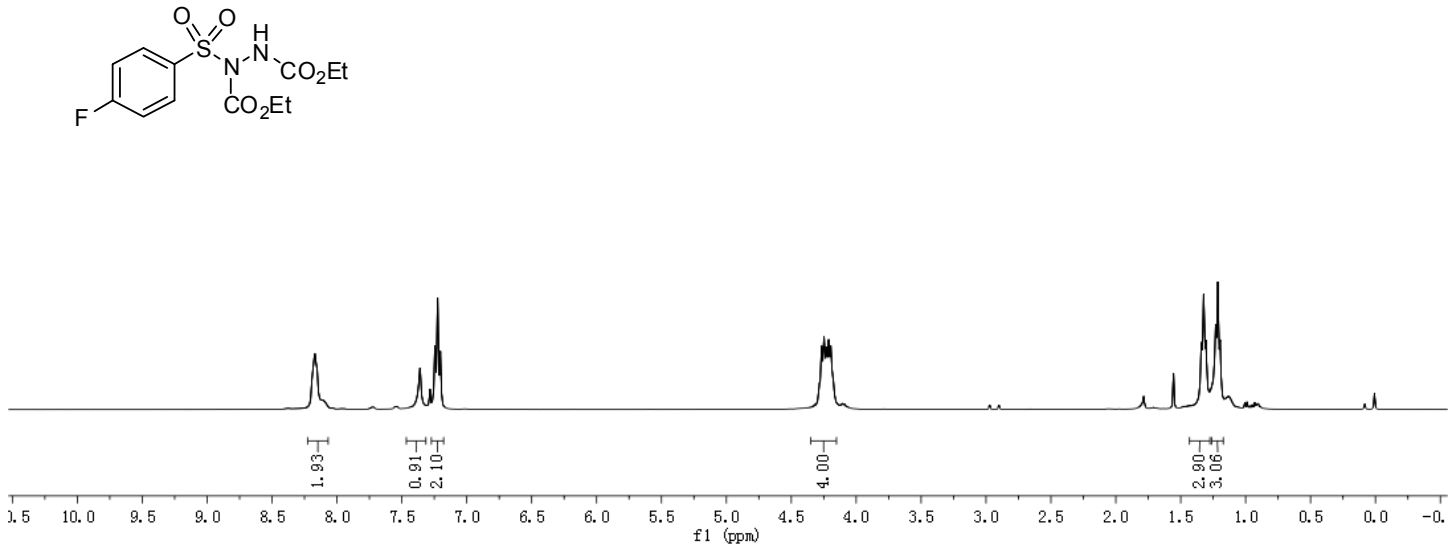

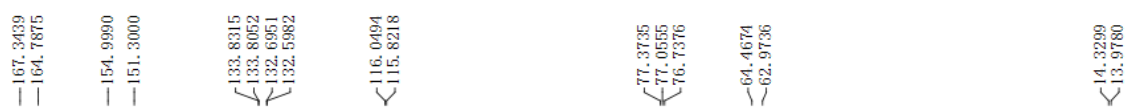
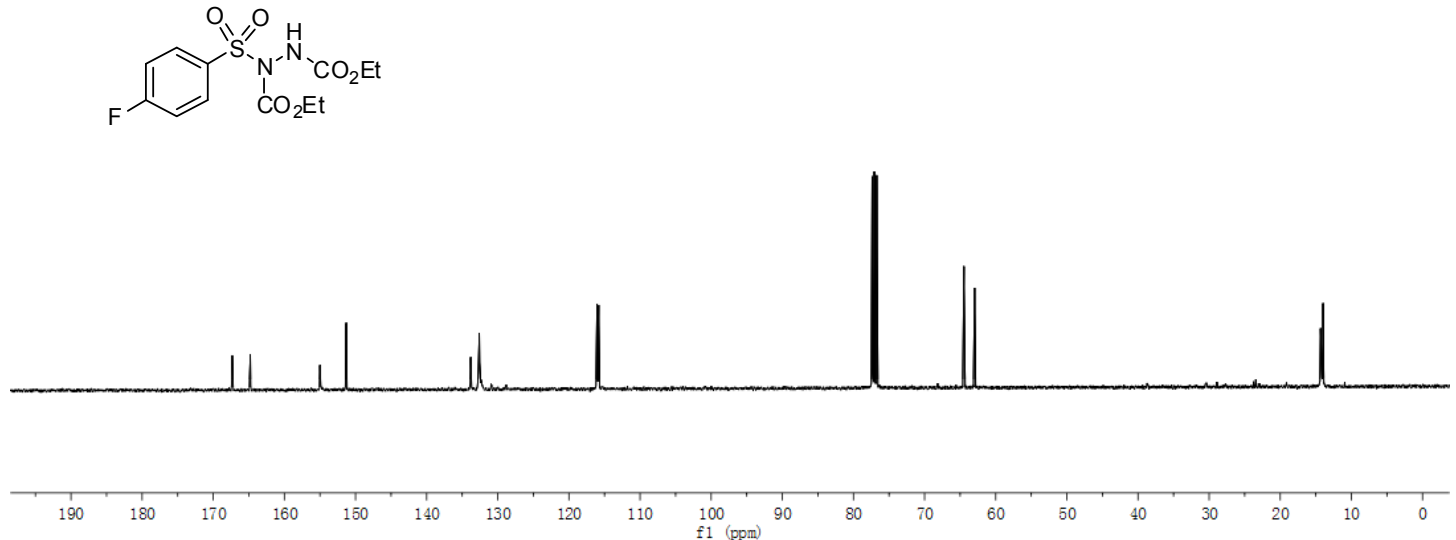


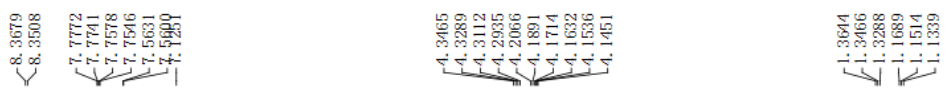

$\mathrm{CO}_{1}^{\mathrm{Br}} \mathrm{CO}_{2}^{\mathrm{O} E t}$

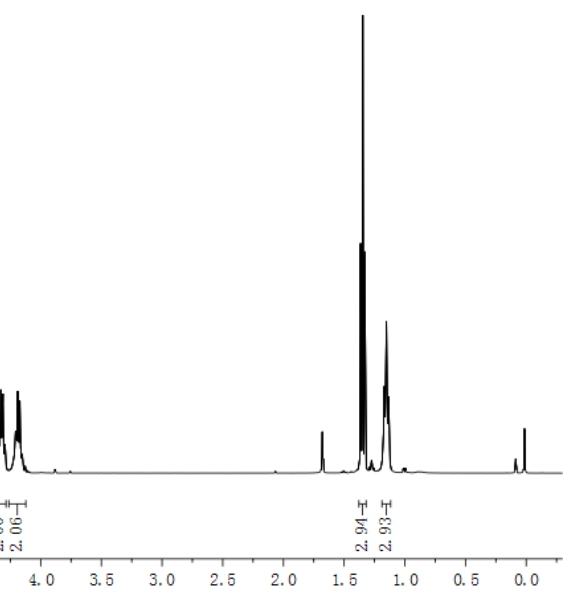

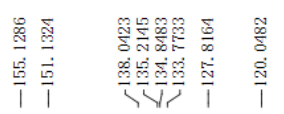

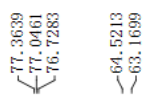
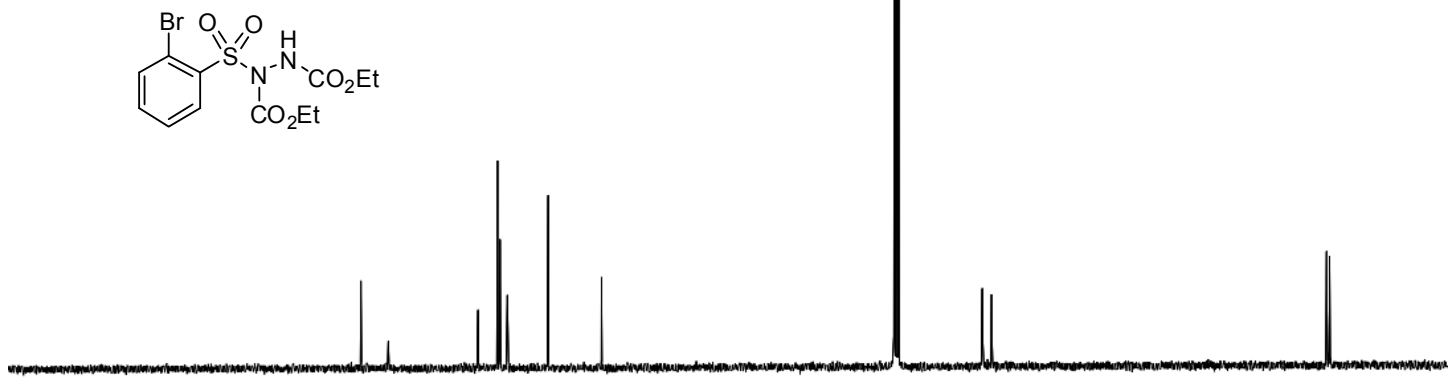

200
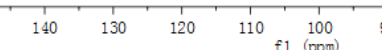


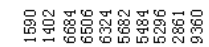

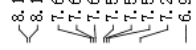

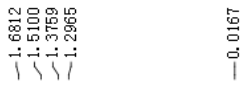
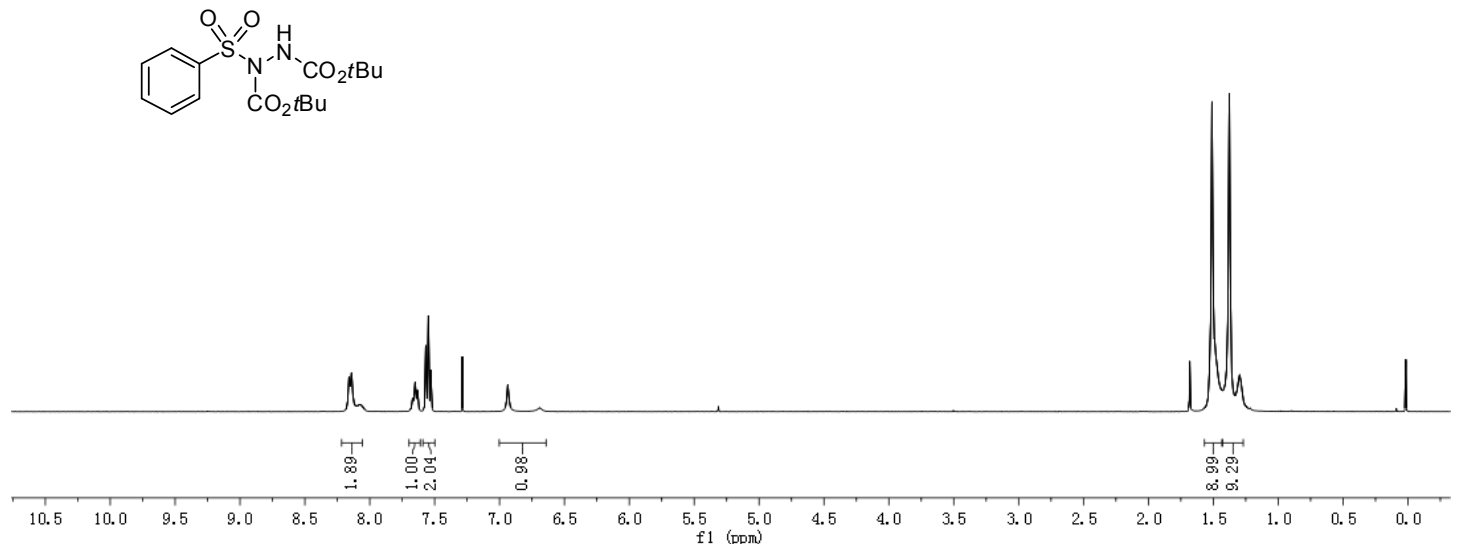

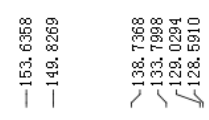

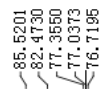

욤율

ฟู่

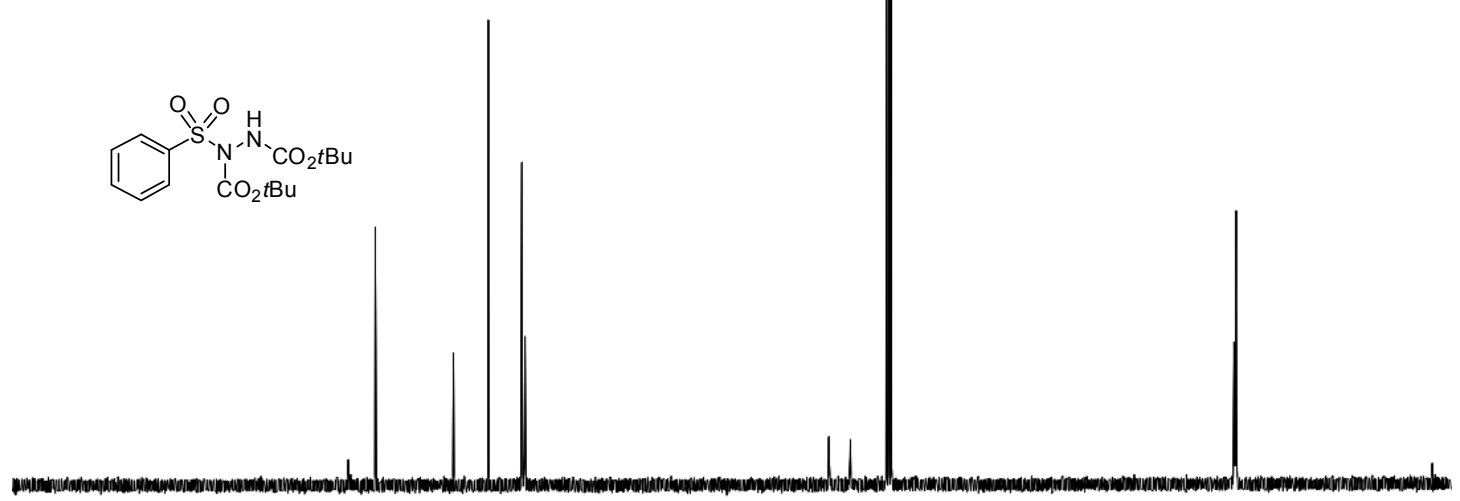

200

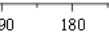

$170 \quad 160$

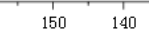

$130 \quad 120$

100
$\mathrm{f} 1(\mathrm{ppm})$

1
$90 \quad 80 \quad 70$
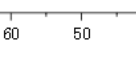

$40 \quad 30$ 

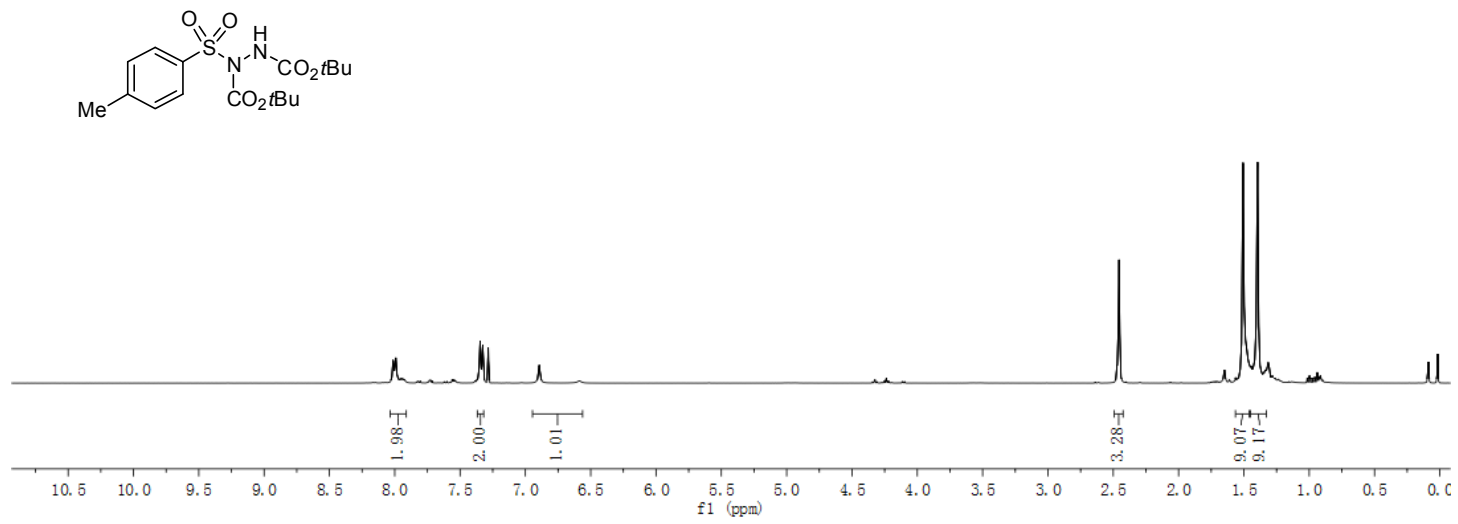

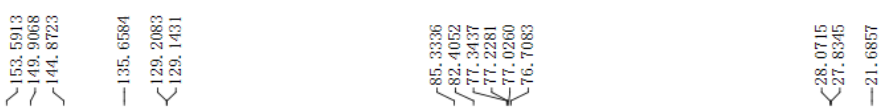
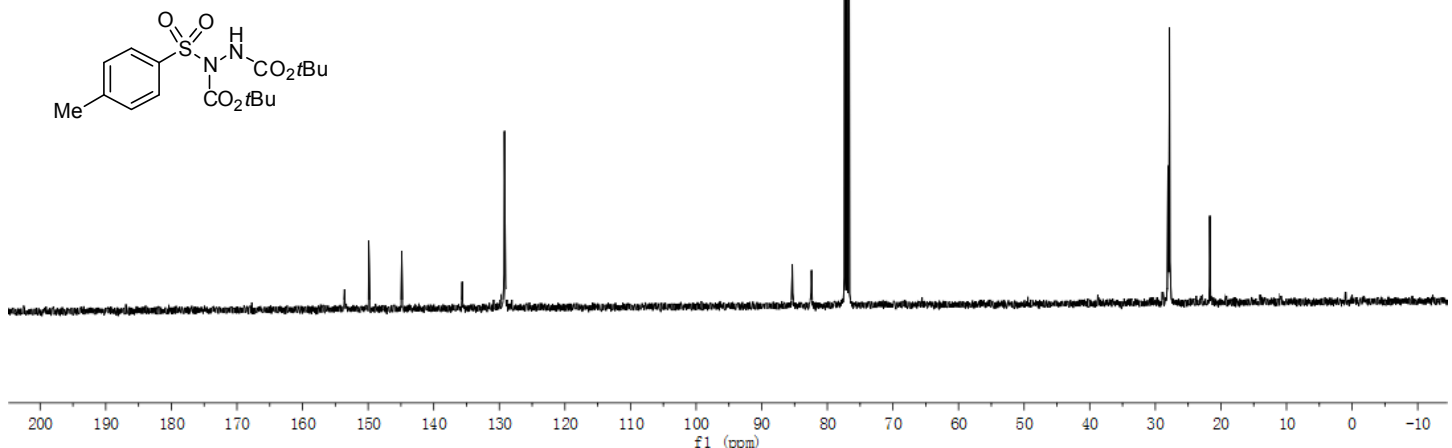

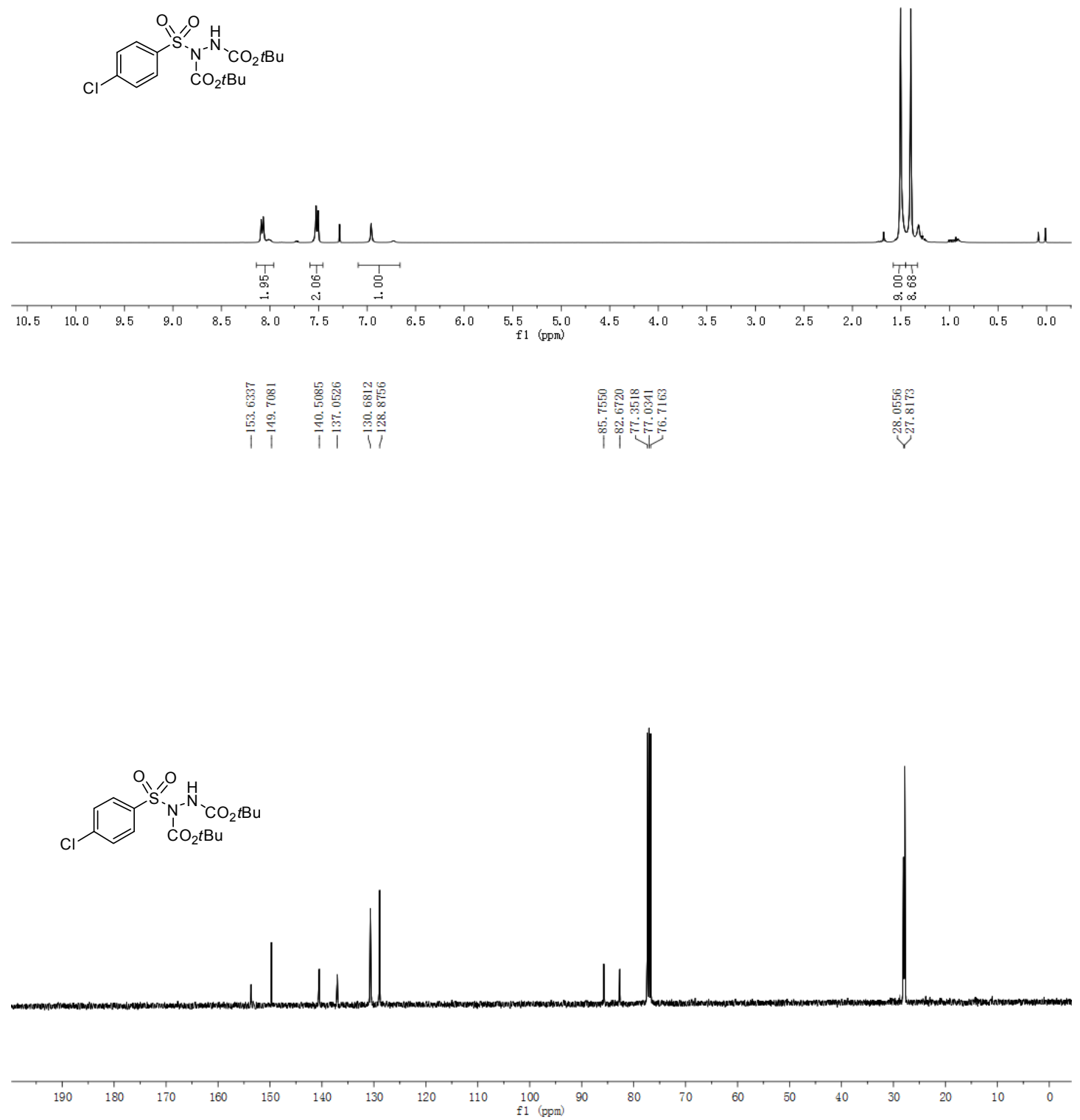

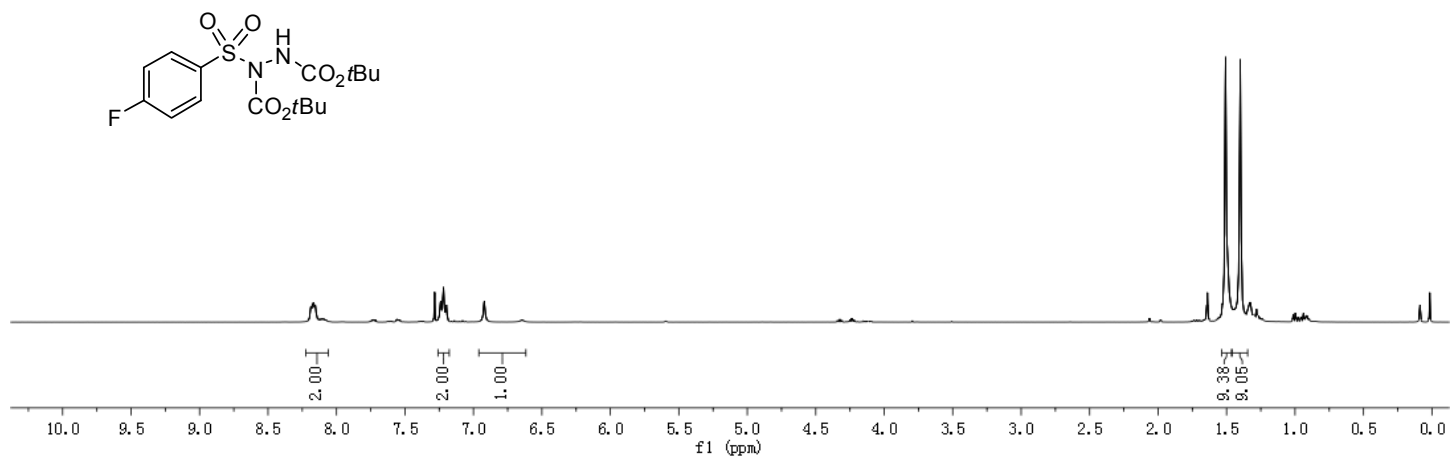

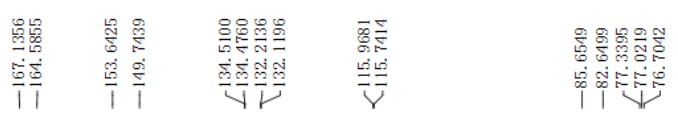
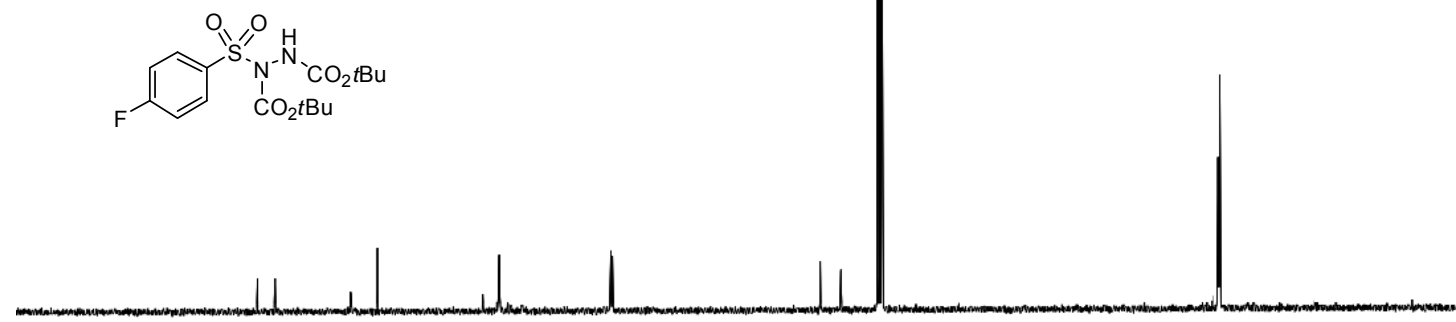

200
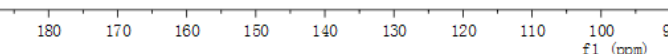

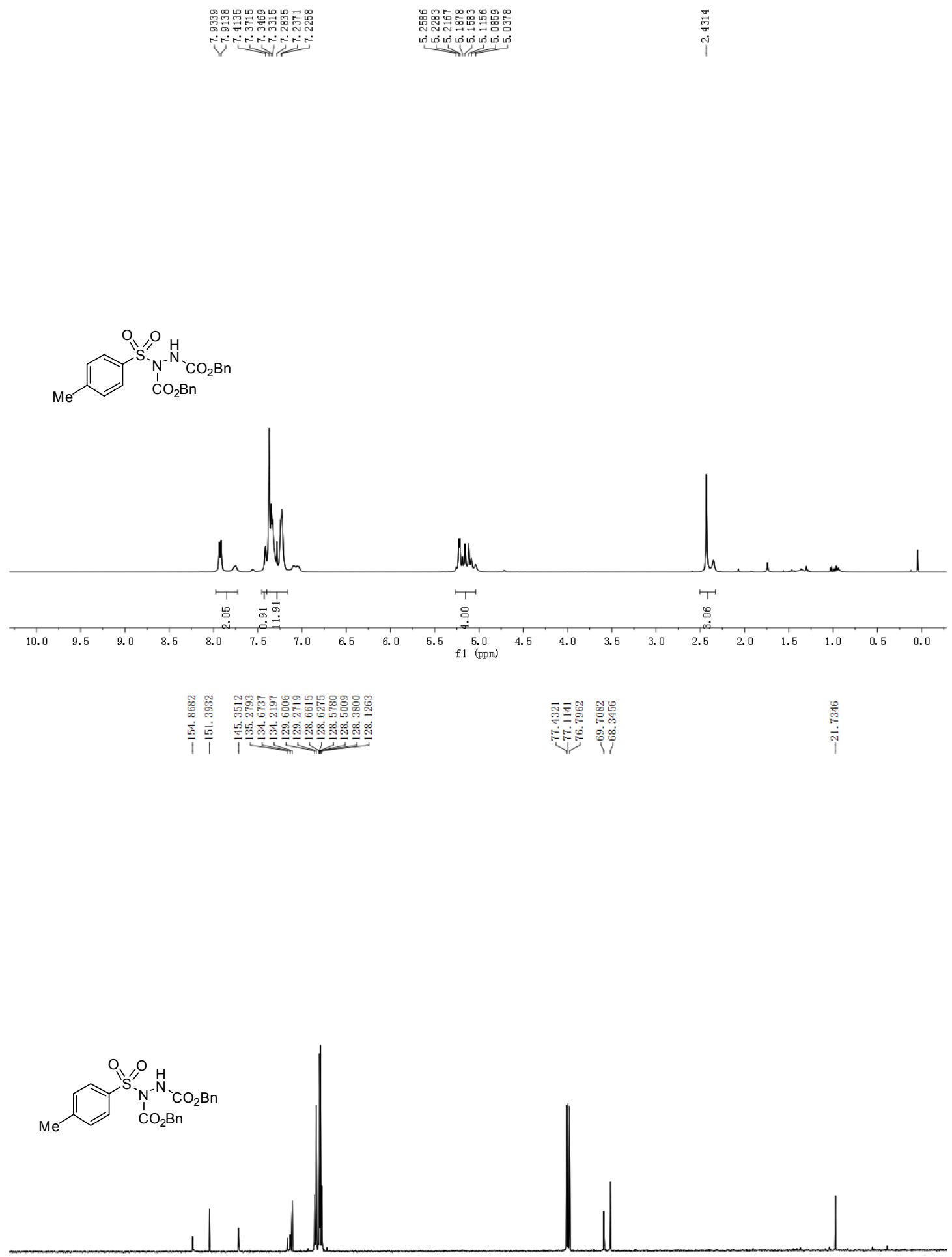

190

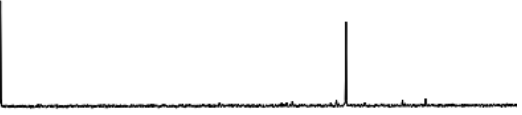




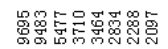

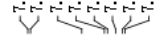

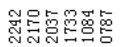

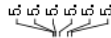
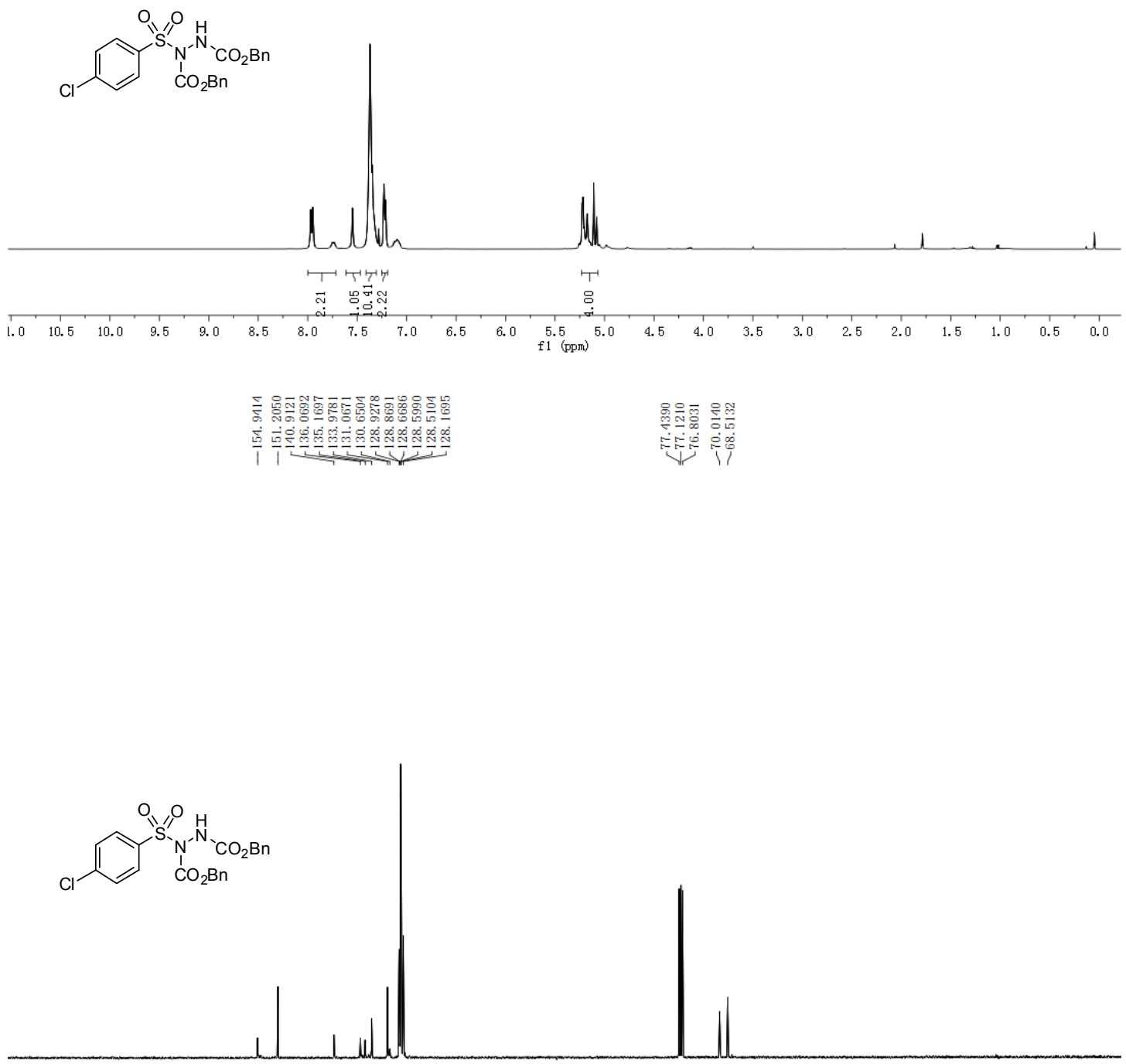

$: 00$
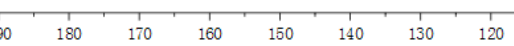

$10 \quad \begin{array}{ll}100 & 1 \\ & \end{array}$ 


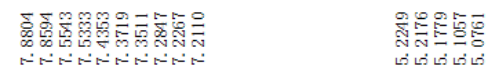

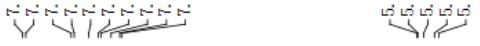

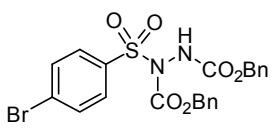

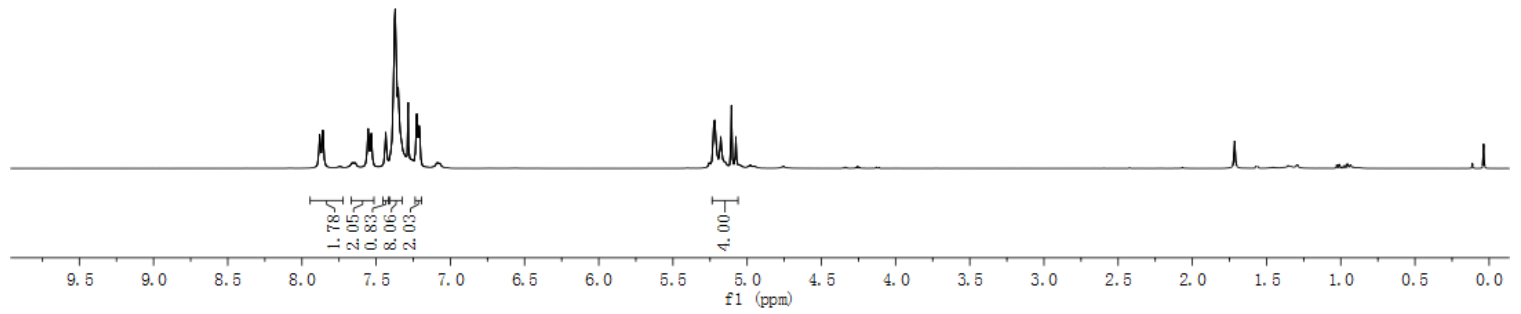

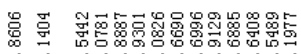

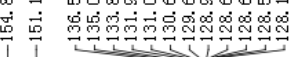

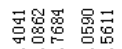

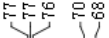

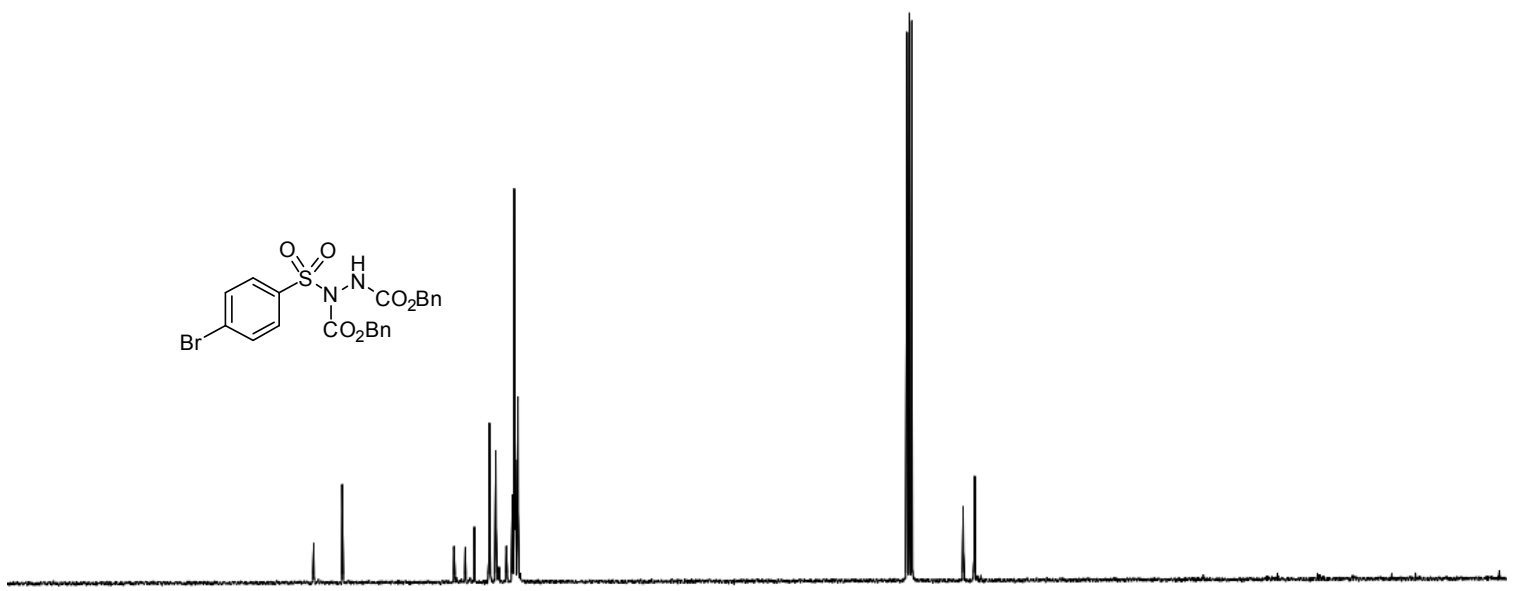

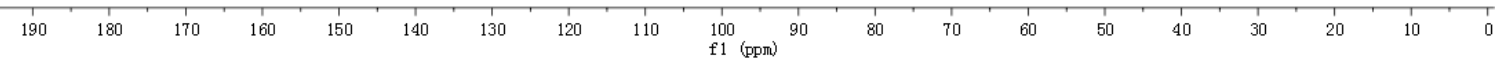


